\title{
Metabolic synthetic lethality by targeting NOP56 and mTOR in KRAS-mutant lung cancer
}

\author{
Zhang Yang ${ }^{1 \dagger}$, Shun-Qing Liang ${ }^{1,2 \dagger}$, Liang Zhao ${ }^{1}$, Haitang Yang ${ }^{1,3}$, Thomas M. Marti ${ }^{1}$, Balazs Hegedüs ${ }^{4}$, \\ Yanyun Gao', Bin Zheng ${ }^{5}$, Chun Chen ${ }^{5}$, Wenxiang Wang ${ }^{6}$, Patrick Dorn' ${ }^{1}$, Gregor J. Kocher ${ }^{1}$, \\ Ralph A. Schmid ${ }^{1 *}$ and Ren-Wang Peng ${ }^{1 *}$ (D)
}

\begin{abstract}
Background: Oncogenic KRAS mutations are prevalent in human cancers, but effective treatment of KRAS-mutant malignancies remains a major challenge in the clinic. Increasing evidence suggests that aberrant metabolism plays a central role in KRAS-driven oncogenic transformation. The aim of this study is to identify selective metabolic dependency induced by mutant KRAS and to exploit it for the treatment of the disease.

Method: We performed an integrated analysis of RNAi- and CRISPR-based functional genomic datasets $(n=5)$ to identify novel genes selectively required for KRAS-mutant cancer. We further screened a customized library of chemical inhibitors for candidates that are synthetic lethal with NOP56 depletion. Functional studies were carried out by genetic knockdown using siRNAs and shRNAs, knockout using CRISPR/Cas9, and/or pharmacological inhibition, followed by cell viability and apoptotic assays. Protein expression was determined by Western blot. Metabolic ROS was measured by flow cytometry-based quantification.
\end{abstract}

Results: We demonstrated that nucleolar protein 5A (NOP56), a core component of small nucleolar ribonucleoprotein complexes (snoRNPs) with an essential role in ribosome biogenesis, confers a metabolic dependency by regulating ROS homeostasis in KRAS-mutant lung cancer cells and that NOP56 depletion causes synthetic lethal susceptibility to inhibition of mTOR. Mechanistically, cancer cells with reduced NOP56 are subjected to higher levels of ROS and rely on mTOR signaling to balance oxidative stress and survive. We also discovered that IRE1 a-mediated unfolded protein response (UPR) regulates this process by activating mTOR through p38 MAPK. Consequently, co-targeting of NOP56 and mTOR profoundly enhances KRAS-mutant tumor cell death in vitro and in vivo.

Conclusions: Our findings reveal a previously unrecognized mechanism in which NOP56 and mTOR cooperate to play a homeostatic role in the response to oxidative stress and suggest a new rationale for the treatment of KRASmutant cancers.

Keywords: KRAS-mutant cancer, NOP56, mTOR, ROS, Synthetic lethal vulnerability

*Correspondence: Ralph.Schmid@insel.ch; Renwang.Peng@insel.ch †Zhang Yang and Shun-Qing Liang contributed equally to this work. ${ }^{1}$ Division of General Thoracic Surgery and Department of BioMedical Research (DBMR), Inselspital, Bern University Hospital, University of Bern, Murtenstrasse 28, 3008 Bern, Switzerland

Full list of author information is available at the end of the article

\section{Background}

Oncogenic mutations in the RAS family (HRAS, KRAS, and $N R A S)$ are the most common genetic alterations across human cancers and occur in approximately $25 \%$ of all tumors (COSMIC; http://cancer.sanger.ac.uk/cosmic). KRAS is the predominant isoform of the RAS family proteins activated by mutations (most frequently at codon 12,13 , and 61 ) in cancers and is responsible for $85 \%$ of all original author(s) and the source, provide a link to the Creative Commons licence, and indicate if changes were made. The images or other third party material in this article are included in the article's Creative Commons licence, unless indicated otherwise in a credit line to the material. If material is not included in the article's Creative Commons licence and your intended use is not permitted by statutory regulation or exceeds the permitted use, you will need to obtain permission directly from the copyright holder. To view a copy of this licence, visit http://creativecommons.org/licenses/by/4.0/. The Creative Commons Public Domain Dedication waiver (http://creativeco mmons.org/publicdomain/zero/1.0/) applies to the data made available in this article, unless otherwise stated in a credit line to the data. 
RAS-driven cancers, particularly pancreatic, colon, and non-small cell lung cancer (NSCLC) [1]. Mutant KRAS is associated with poor prognosis and treatment resistance. However, unlike NSCLC with less frequent oncogenic drivers (e.g., EGFR, ALK, MET1, and ROS1) that respond significantly to selective kinase inhibitors [2], effective therapies specifically targeting $K R A S$-mutant cancers remains a challenge $[2,3]$. Despite recent progress of immune checkpoint inhibitors of programmed death 1 (PD1) and the ligand PD-L1 in treating NSCLC, they fail to discriminate KRAS-mutant from other NSCLC [4]. Covalent KRAS inhibitors have demonstrated promise in preclinical models, but they are only effective for a specific KRAS-G12C mutant allele and additional agents are needed to optimize the anticancer efficacy [5-7]. Targeting KRAS downstream effectors, such as the mitogenactivated protein kinase (MAPK) RAF/MEK/ERK, has been widely pursued, but the pleiotropic nature and complex interplay among individual signaling cascades and toxicity ensuing from sustained inhibition of multiple KRAS effector pathways has hindered the translational potential of the strategy $[8,9]$. Consequently, identification of new targets for innovative treatment strategies tailored to KRAS-mutant cancers still represents a pressing need [3].

The concept to target KRAS synthetic lethality, premised by the notion that oncogenic KRAS signaling fuels a unique cell state, manifested by adaptation to oncogenic stress and transcriptional, translational and metabolic reprogramming, and that interfering with this KRASdriven cell state may result in selective cytotoxicity for $K R A S$-mutant cancer, provides an alternative strategy for treating KRAS-driven cancers $[10,11]$. Indeed, exploiting cancer cell vulnerabilities contextually induced by mutant KRAS, in particularly the mechanisms critical for surveillance of oncogene-dependent cellular stresses (genotoxic, proteotoxic, and metabolic) that are permissive for strong oncogenic signaling, has not only provided promising therapeutic avenues but also a wealth of information on the fundamental principles of KRAS-induced tumorigenicity [12-14]. Activating KRAS mutations deregulate mitosis, nuclear export, redox, and mitochondrial activity, and KRAS-mutant cancer cells have consequently been shown to have a greater dependency on the functions of non-oncogenes [e.g., PLK1, XPO1, and MRPL52 (a component of the mitochondrial large ribosomal subunit)] that play critical roles in their respective processes [12-16], suggesting that targeting non-oncogene addiction is an attractive approach for the treatment of KRAS-mutant cancer [17, 18].

NOP56 (nucleolar protein 5A or NOL5A) is a ribonuclear protein, which, together with fibrillarin (FBL), NOP58 (nucleolar protein 58), and nonhistone chromosome protein 2-like 1 (NHP2L1 or SNU13p, $15.5 \mathrm{kDa}$ ), forms the core protein set of box C/D small nucleolar ribonucleoprotein complexes (snoRNPs) that play an essential role in ribosome assembly by methylating rRNA at the 2'-O-ribose and modulating ribosomal RNA (rRNA) processing [19, 20]. Recent evidence suggests that NOP56 and the other snoRNPs are the novel group of nucleolar proteins that promote cell transformation and tumorigenesis [21, 22]. Indeed, ribosome biogenesis is the only cellular process in which a large number of genes harbor evolutionarily conserved MYCbinding sites [21]. NOP56 is overexpressed in Burkitt's lymphoma and other cancers and serves as a marker of poor prognosis [23]. In particular, NOP56 is required for MYC-induced cell transformation and tumor growth in Burkitt's lymphoma [21]. NOP56 may also have extraribosomal functions that remain to be discovered. Nevertheless, the activity of snoRNPs in oncogenic transformation suggests that they are promising therapeutic targets for cancer treatment [24, 25].

In this study, we reported an unexpected function of NOP56 in metabolic stress response and a previously unrecognized metabolic synthetic lethality by targeting NOP56 and mTOR in KRAS-mutant cancers. Based on integrated analyses of RNAi- and CRISPR-mediated functional genomics $[12,16,26]$, we identified NOP56 as a novel metabolic dependency of KRAS-mutant cancer by regulating homeostasis of reactive oxygen species (ROS) that plays a well-established role in mutant KRAS-induced tumorigenesis [27-30]. Depletion of NOP56 impairs the response to oxidative stress, which renders $K R A S$-mutant cancer cells highly dependent on mTOR signaling for survival and particularly vulnerable to mTOR inhibition. Consequently, co-targeting NOP56 and mTOR enhances apoptotic death of KRAS-mutant lung cancer cells in vitro and in vivo. We further delineated that mTOR activation upon NOP56 depletion is mediated by IRE1 $\alpha$-mediated unfolded protein response (UPR). These results uncover a previously unknown mechanism by which NOP56 cooperates with UPR and mTOR to regulate metabolic stress and a novel synthetic lethal strategy for the treatment of KRAS-mutant cancers.

\section{Materials and methods \\ Cell culture and reagents}

Cancer cell lines used in this study (Table S1) were obtained from American Type Culture Collection (ATCC, Manassas, VA, USA). Cells were cultured in RPMI-1640 medium or Medium 199 (Cat. \#8758 and \#4540; Sigma-Aldrich, St. Louis, MO, USA) supplemented with $10 \%$ fetal bovine serum/FBS (Cat. \#10270-106; Life Technologies, Grand Island, NY, USA) 
and $1 \%$ penicillin/streptomycin solution (Cat. \#P0781, Sigma-Aldrich). The cells were authenticated by DNA fingerprinting and confirmed free from mycoplasma contamination (Microsynth, Bern, Switzerland). All inhibitors used in this study were listed in Table S2.

PF139 and PF563 lung cancer cells were established from lung adenocarcinoma malignant pleural effusion and pleural carcinosis specimens of a 67 year-old female patient and a 75 year-old male patient, respectively, at the time of diagnosis prior to any treatment [31]. Authentication was performed by SNP based cell identification (Multiplexion, Heidelberg, Germany).

\section{Cell viability and clonogenic survival assay}

Lung cancer cells seeded in 96-well plates (2500 cells/ well) were dosed $24 \mathrm{~h}$ later with different inhibitors for $72 \mathrm{~h}$. Cell viability was determined by PrestoBlue (PB) Cell Viability Reagent (ThermoFisher Scientific) by following the manufacturer's instructions $[14,31]$. The PB reagent was added into media directly (1:10 dilution) and incubated for $30 \mathrm{~min}-2 \mathrm{~h}$ and then the fluorescence was read (excitation $570 \mathrm{~nm}$; emission $600 \mathrm{~nm}$ ) at recommended time of incubation. The efficacy of drugs on cell growth was normalized to untreated control. Each data point was generated in triplicate and each experiment was done three times $(n=3)$. Best-fit curve was generated in GraphPad Prism [ $(\log$ (inhibitor) vs response (-variable slope four parameters)]. Error bars are mean \pm SD. The combination index (CI) was calculated by ComboSyn software (ComboSyn Inc., http://www.combosyn.com/).

Clonogenic assay was done as we described previously [14, 31-33]. In brief, cells seeded in 6-well plates (3000 cells/well) were dosed $24 \mathrm{~h}$ later and continually treated with rapamycin for 7 days (refresh drugs every 3 days), the resulting colonies were stained with crystal violet ( $0.5 \%$ dissolved in $25 \%$ methanol).

\section{Apoptosis assays}

Lung cancer cells were treated for $72 \mathrm{~h}$ with vehicle or rapamycin. After treatment, cells in the supernatant and adherent to plates were collected, washed with PBS and pooled before suspended in binding buffer and stained with the Annexin V Apoptosis Detection Kit -FITC (Cat. \#88-8005; Thermo Fisher Scientific, Waltham, MA, USA) according to the manufacturer's instructions. Flow cytometry analysis was performed on a BD Biosciences LSRII flow cytometer.

\section{Gene silencing by small interfering (siRNA), short hairpin RNAs (shRNA) and single-guide RNAs (sgRNA)}

Transient knockdowns were mediated by siRNAs. Cells cultured in triplicate at $50-70 \%$ confluency were transfected using SiTran1.0 (TT300001; Origene
Technologies, Rockville, MD, USA) according to the manufacturer's protocol. NOP56 (CAT\#: SR307156), EIF4E (CAT\#: SR320018), RPS6 (CAT\#: SR304160), RAPTOR (CAT\#: SR324724), and RICTOR (CAT\#: SR326062) were knocked down by specific pooled siRNA duplexes purchased from OriGene Technologies, with control siRNA Duplex as a negative control.

Stable knockdown of NOP56 was achieved via lentiviral delivery of NOP56 Human shRNA Plasmid Kit (SHCLND_006392, MERCK). A scramble shRNA was used as a control. Lentiviral particles were generated and cells infected according to the protocol from Broad Institute. The supernatant containing lentiviruses was collected, filtered through $0.45 \mu \mathrm{M}$ filters, and stored in aliquots at $-80^{\circ} \mathrm{C}$, or immediately used to infect recipient cells. After infection, cells were selected in puromycin $(1.5 \mu \mathrm{g} / \mathrm{ml})$ and further passaged in culture for functional assays. NOP56 knockout was performed via a CRISPR/ Cas9 and non-homology mediated approach using the NOL5A (NOP56) Human Gene Knockout Kit (CAT\#: KN411153; OriGene Technologies) according to the manufacturer's protocol.

\section{Quantitative real-time PCR (qRT-PCR)}

Total RNA was isolated and purified using RNeasy Mini Kit (Qiagen, Hilden, Germany). Complementary DNA was synthesized by the High capacity cDNA reverse transcription kit (Applied Biosystems, Foster City, CA, USA) according to manufacturer's instructions. Real time PCR was performed in triplicate on a 7500 Fast RealTime PCR System (Applied Biosystems) using TaqMan primer/ probes (Applied Biosystems): HSPA5, Hs00607129_gH; ERN1, Hs00980095_m1; EIF2AK3, Hs00984003_m1; ATF4, Hs00909569_g1; DDIT3, Hs00358796_g1, with GAPDH (Hs02786624_g1) and ACTB (Hs01060665_g1) used as endogenous normalization controls.

\section{Immunoblotting, immunohistochemistry and immunofluorescence}

Cell lysates were prepared and western blot analysis was performed as described [14, 31]. In brief, equal amounts of protein lysates resolved by SDS-PAGE (Cat. \#4561033; Bio-Rad Laboratories, Hercules, CA, USA) and transferred onto nitrocellulose membranes (Cat. \#170-4158; Bio-Rad). Membranes were then blocked with blocking buffer (Cat. \#927-4000; Li-COR Biosciences, Bad Homburg, Germany) for $1 \mathrm{~h}$ at room temperature (RT) and incubated with appropriate primary antibodies overnight at $4{ }^{\circ} \mathrm{C}$ (Table S3). IRDye 680LT-conjugated goat anti-mouse IgG (Cat. \#926-68,020) and IRDye 800CWconjugated goat anti-rabbit IgG (Cat. \#926-32,211) from Li-COR Biosciences were used at 1:5000 dilutions. Finally, signals of membrane-bound secondary antibodies 
were imaged using the Odyssey Infrared Imaging System (Li-COR Biosciences).

For immunofluorescence, tumor cells grown on polylysine-treated coverslides were fixed with $4 \%$ paraformaldehyde for $15 \mathrm{~min}$ at RT and permeabilized with cold methanol $\left(-20^{\circ} \mathrm{C}\right)$ for 5 min or with $0.1 \%$ Triton X-100/ PBS at RT for $15 \mathrm{~min}$ before incubated overnight at $4{ }^{\circ} \mathrm{C}$ with primary antibodies (Table S3). The cells were incubated for $1 \mathrm{~h}$ at RT with Alexa Fluor 647 goat anti-mouse IgG (Cat. \#A21236) or Alexa Fluor 488 goat anti-Rabbit IgG (Cat. \#A11034) from Invitrogen (Eugene, OR, USA). Nuclei were counterstained by $4^{\prime}, 6$-diamidino-2-phenylindole. Images were acquired on a ZEISS Axioplan 2 imaging microscope (Carl Zeiss MicroImaging, Göttingen, Germany) and processed using Adobe Photoshop CS6 v.13 (Adobe Systems, San Jose, CA, USA).

Immunohistochemical study was performed as we described previously [31, 32]. In brief, surgically removed xenograft tumors were formalin-fixed and paraffinembedded (FFPE). FFPE tumors were sectioned at $4 \mu \mathrm{m}$, deparaffinized, rehydrated and subsequently stained with hematoxylin and eosin (H\&E) and appropriate antibodies (Table S3) using the automated system BOND RX (Leica Biosystems, Newcastle, UK). Visualization was performed using the Bond Polymer Refine Detection kit (Leica Biosystems) as instructed by the manufacturer. Images were acquired using PANNORAMIC ${ }^{\circledR}$ whole slide scanners, processed using Case Viewer (3DHISTECH Ltd.). The staining intensities of the whole slide (two tumors/group) were quantified by QuPath software.

\section{In vivo mouse study}

Mouse studies were conducted in accordance with Institutional Animal Care and Ethical Committee-approved animal guidelines and protocols. All mouse experiments were performed in age- and gender-matched NSG (NOD-scid IL2R ${ }^{\text {null }}$ ) as we previously described $[31,32]$. Tumor cells in DMEM (H460-shScrambled or H460-shNOP56) 1:1 mixed with BD Matrigel Basement Membrane Matrix (Cat. \#356231; Corning, NY, USA) were subcutaneously inoculated in left and right flanks $\left(0.5 \times 10^{6} /\right.$ injection $)$. When tumors were palpable, mice were randomly assigned to treatment groups: 1$)$ control; 2) rapamycin $(0.1 \mathrm{mg} / \mathrm{kg}$, i.p, 5 days/week) for 5 weeks. Tumors were measured every 3 days, with their size calculated as follows: (length $\times$ width $^{2}$ )/2. For survival analysis, the mice were closely monitored on a daily basis, and the size of tumors was measured with a caliper every $4-5$ days. Mice were sacrificed when the tumor volume reached $1500 \mathrm{~mm}^{3}$.

\section{Public databases}

To identify synthetic lethal targets in KRAS-mutant cancers, we interrogated functional genomics dataset of CRISPR/Cas9 knockout and RNAi/shRNA knockdown screens from published studies: whole genome RNAi screens in DLD-1 colon cancer cells [12] and in KRASmutant lung cancer cells (H2122, H2009, HCC44, H460, H1155) [16], genome-wide CRISPR/Cas9 loss of function screens in KRAS-mutant leukemia cells (PL-21, SKM1, NB4) [26]. To minimize the effects of cancer lineage and histological subtype, we selected DLD-1 (colon), H460 (large cell lung carcinoma), H2122 (lung adenocarcinoma), SKM-1 (without PML-RARA fusion) and NB4 (PML-RARA) for further analysis, which identified a number of common candidates $(n=21)$ as KRAS synthetic lethal partners (Fig. 1).

Interrogation of publicly available datasets was performed as we have described [14, 31]. Specifically, transcriptomic data of lung, pancreatic and colon cancer were obtained from the Cancer Genome Atlas (TCGA) (https://portal.gdc.cancer.gov/projects/TCGA). Gene set enrichment analysis (GSEA) was performed by using GSEA software. The transcriptomic dataset (GSE15212) used for GSEA was derived from KRAS-mutant colon cancer cell line (SW480) treated with NOP56-specific siRNAs and downloaded from the Gene Expression Omnibus (GEO) database. For survival analysis, transcriptomic gene expression and corresponding survival data were extracted and analyzed by using the "maxstat", "survival", and "survminer" packages in R software

\footnotetext{
(See figure on next page.)

Fig. 1 NOP56 confers a metabolic dependency in KRAS-mutant cancers. A, Venn diagram showing common essential genes in KRAS-mutant cancer cells. Data are based on the published studies, with the 21 common genes listed on the right. B, Network analysis of the 21 common genes by STRING. C, NOP56 mRNA expression in KRAS-mutant lung cancer (LC), pancreatic cancer (PC) and colon cancer (CC) versus KRAS-wild-type (WT) cancers in patient samples from TCGA. D, Prognostic values of NOP56 expression across TCGA lung adenocarcinoma (left), pancreatic cancer (middle) and colon cancer (right) cohorts harboring KRAS mutations. Kaplan-Meier survival analyses were stratified by the optimal cut-off value of NOP56 mRNA levels. E, Gene set enrichment analysis (GSEA) revealed significant enrichment of oxidative phosphorylation and ROS pathway gene signatures in NOP56-depleted KRAS-mutant cancer cells (SW480). The GEO dataset GSE15212 was used for GSEA. F, H358 and H460 cells transfected with NOP56-specific or control siRNAs were treated (72 h post-transfection) with $300 \mathrm{\mu M} \mathrm{H}_{2} \mathrm{O}_{2}$ for $6 \mathrm{~h}$, followed by incubation with $\mathrm{H} 2 \mathrm{DCFDA}$ for $30 \mathrm{~min}$, and analyzed by flow cytometry. Quantification of relative ROS levels was shown in the right. Data are shown as the mean \pm SD $(n=3) .{ }^{*} P<0.05$, ${ }^{* *} P<0.001,{ }^{* * *} P<0.0001$ by two-way ANOVA with Tukey's multiple comparisons test. G, H358 and H460 cells transfected with NOP56-specific or control siRNAs were subsequently ( $72 \mathrm{~h}$ post transfection) treated with vehicle (DMSO) or $300 \mu \mathrm{M} \mathrm{H}_{2} \mathrm{O}_{2}$ for $6 \mathrm{~h}$ before apoptotic assay. Data were shown as mean \pm SD $(n=3) . * P<0.05$ and ${ }^{* * *} P<0.001$ by two-way ANOVA with Tukey's multiple comparisons test
} 
A

C

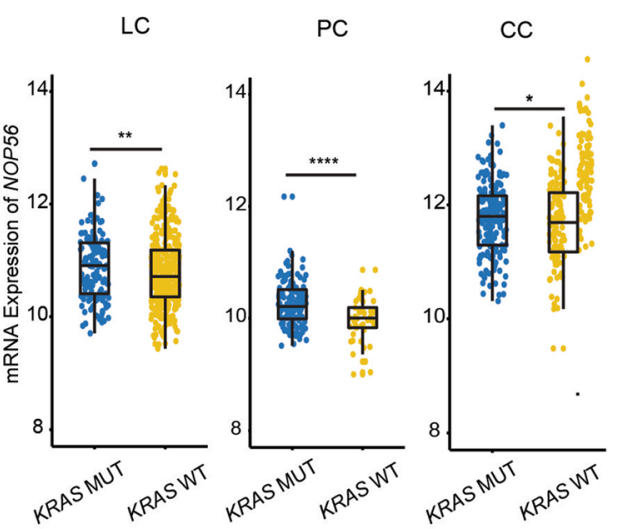

E OXIDATIVE_PHSPHORYLATION

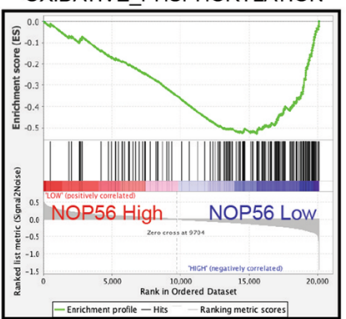

HALLMARK ROS PATHWAY

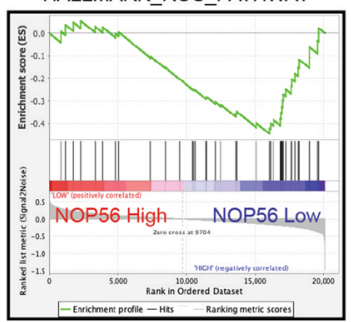

F
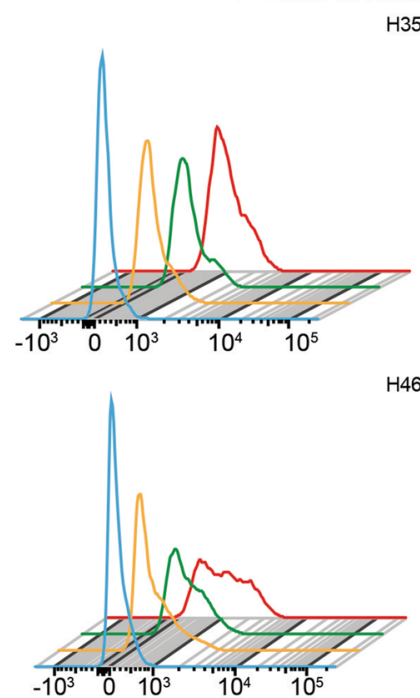

B

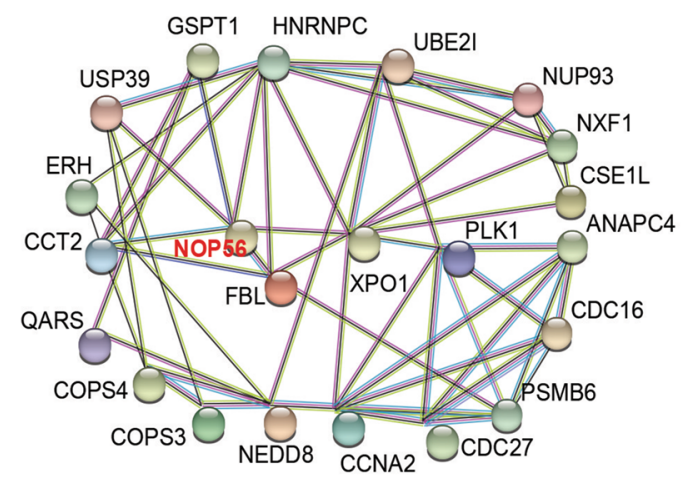

$D_{\text {g }}$

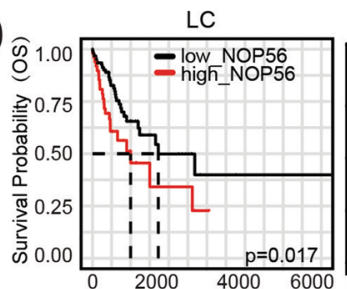

PC

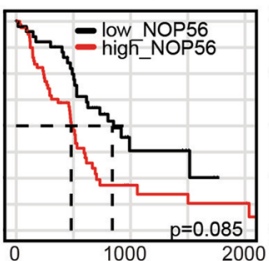

CC
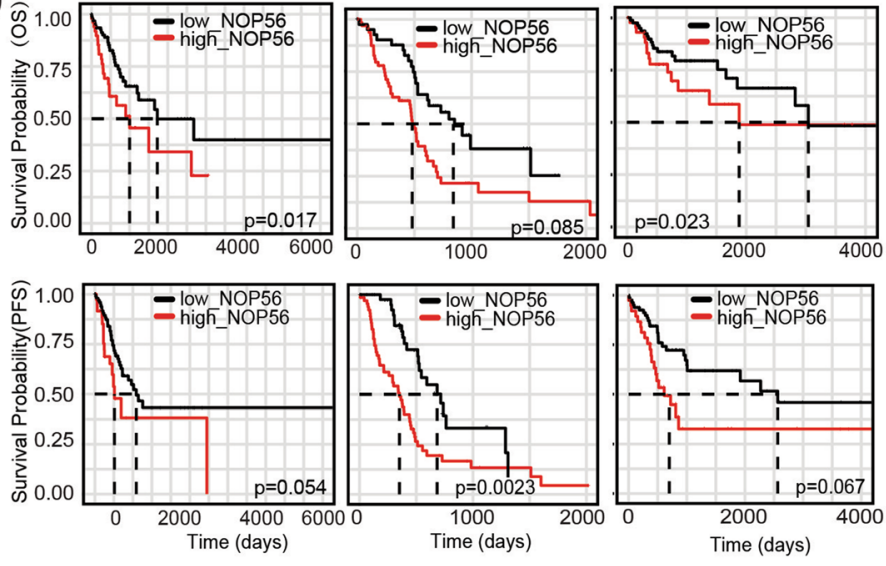

$\square$ siControl+DMSO $\square$ siControl+H2O2

$\square$ siNOP56+H2O2

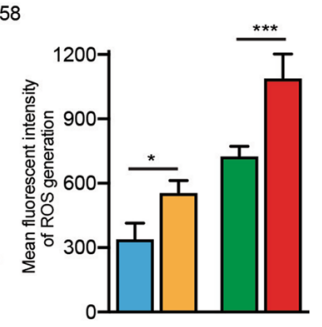

G
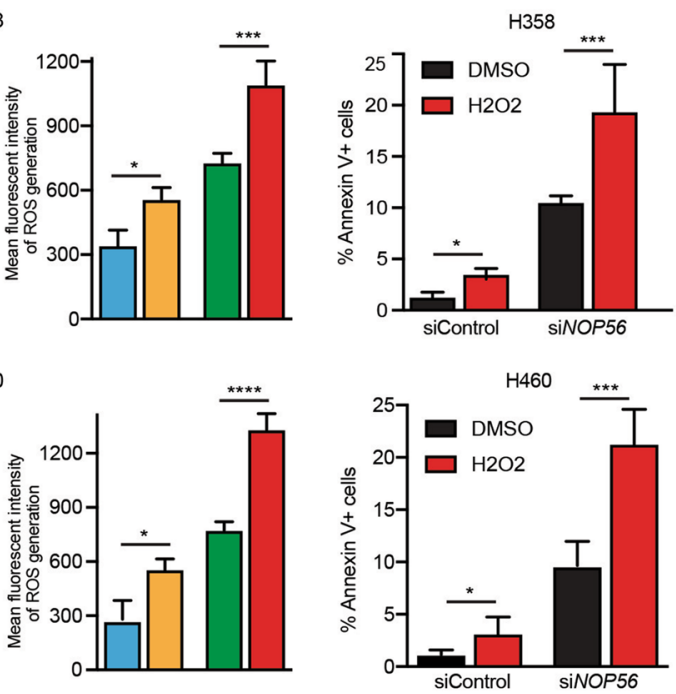

Fig. 1 (See legend on previous page.) 
(version 3.6.0). Patients were divided into two groups (high_NOP56 versus low_ NOP56) based on the optimal cutoff value of NOP56 transcripts across all patients to plot the Kaplan-Meier survival curves.

For correlative analysis of NOP56 expression with sensitivity $\left(\mathrm{IC}_{50}\right)$ to mTOR inhibitors, gene expression data and drug response profiles were downloaded from Cancer Cell Line Encyclopedia (CCLE) and Genomics of Drug Sensitivity in Cancer (GDSC) databases, respectively. Correlation analysis was performed using $\mathrm{R}$ software (version 3.6.0).

\section{Statistical analysis}

Statistical analyses were performed using GraphPad Prism 7.01 (GraphPad Software Inc., San Diego, CA, USA) unless otherwise indicated. In all studies, data represent biological replicates $(\mathrm{n})$ and are depicted as mean values \pm SD or mean values \pm SEM as indicated in the figure legends. In all analyses, $P$ values less than 0.05 were considered statistically significant. For the survival analysis, patients were grouped by gene expression, where 'high' and 'low' expression groups were stratified by the optimal cut-off value.

\section{Results}

NOP56 confers a metabolic dependency by regulating ROS homeostasis in KRAS-mutant lung cancer

To identify therapeutic vulnerabilities in KRAS-mutant cancers, we performed integrated analysis of shRNA- and CRISPR-based functional genomics of previously published studies [12, 16, 26]. To minimize lineage-specific effects, we analyzed whole-genome dropout screen dataset in KRAS-mutant lung (H460, H2122), colon (DLD-1), acute promyelocytic leukemia (NB4) and acute myeloid leukemia (SKM-1) cancer cells, which identified 21 common genes whose loss of function is synthetic lethal with mutant KRAS alleles in distinct cancer lineages (Fig. 1A; Table S4). The protein products of these genes fall into several functional categories, with FBL, NOP56, PLK1 and XPO1 as a core set based on their interaction network (Fig. 1B). Remarkably, PLK1 and XPO1 have been reported to be selectively required for KRAS-mutant cancers by counteracting mitotic and nuclear export stress associated with KRAS-induced tumorigenesis [12, 16], and our recent study has implicated PLK1 in metabolic stress response of KRAS-mutant cancers [14]. FBL has also been assigned as a promising target in cancers [34, 35], suggesting the power of functional genomics in identifying oncogene-specific vulnerabilities and the accountability of our analyses. In the present study, we investigated the function of NOP56 in KRAS-mutant cancers.
Our investigations began with NOP56 knockdown using small interfering RNAs (siRNAs), which revealed that downregulation of NOP56 significantly inhibited the proliferation of numerous KRAS-mutant lung $(\mathrm{H} 358$, H460, A549, PF563, PF139), pancreatic (MIAPaCa, HPAF-II) and colon (HCT-116, DLD-1) cancer cells, which differ not only in tumor lineages and histological subtypes but also in KRAS mutations, e.g., G12C, G12D, $\mathrm{Q} 61 \mathrm{H}$, etc. (Fig. S1A, B; Table S1). Notably, NOP56 silencing also inhibited NRAS-mutant lung cancer H1299 cells, although the effects on EGFR-mutant (EBC-1) or FGFR1-amplified (H520) lung cancer cells were negligible (Fig. S1A, B). Supporting these observations, $K R A S$-mutant lung, pancreatic and colon cancer showed significantly higher expression of NOP56 than KRAS-WT tumors (Fig. 1C) and patients with KRAS-mutant lung adenocarcinoma, pancreatic and colon cancer characterized by a higher NOP56 level are associated with significantly shorter survival (Fig. 1D). In contrast, NOP56 expression is not a prognostic marker for $K R A S$-mutant lung, pancreatic and colon cancers (Fig. S1C). These results indicate a unique function for NOP56 in KRASmutant cancers.

To explore NOP56 functions in KRAS-mutant malignancies, we profiled the transcriptomic gene expression data of a previous study [36], whereby NOP56 in KRAS-mutant colon cancer cells (SW480) was silenced by siRNAs. Our analysis revealed that high expression of NOP56 was positively correlated with the gene signature of KRAS signaling (Fig. S1D), in line with the above results (Fig. 1A-D; Fig. S1A, B), and that, importantly, siRNA-mediated NOP56 knockdown led to significant enrichment of the gene sets involved in ROS pathway (consisting of 49 genes upregulated by ROS) and oxidative phosphorylation (a set of 200 genes encoding proteins involved in oxidative phosphorylation), the latter representing a major source of ROS production (Fig. 1E), suggesting a possible role for NOP56 in the suppression of metabolic ROS that is critical for KRAS-induced tumorigenesis [27-30]. Supporting this notion, NOP56 knockdown (KD) by siRNAs significantly upregulated ROS in $\mathrm{H} 358$ and $\mathrm{H} 460$ cells, and $\mathrm{H}_{2} \mathrm{O}_{2}$ treatment, which elevated the already high level of oxidative stress, provoked significantly greater apoptosis in NOP56 KD H358 and $\mathrm{H} 460$ cells than the control counterparts (Fig. 1F, G). These results uncover NOP56 as a metabolic dependency in KRAS-mutant cancer by exerting a previously unrecognized role in the surveillance of oxidative stress.

\section{NOP56 suppression evokes IRE1 a-mediated UPR to mitigate oxidative stress}

Next, we investigated the mechanism that $K R A S$-mutant cancer cells utilize to orchestrate cytotoxic ROS upon 
NOP56 depletion. GSEA of transcriptomic dataset [36] revealed that NOP56 knockdown significantly enriched the genes involved in the unfolded protein response/UPR (a set of 113 genes upregulated during UPR) in KRASmutant cancer cells (Fig. 2A), suggesting that tumor cells might engage the UPR to protect from NOP56 KDinduced surge of cytotoxic ROS. To test this possibility, we knocked down NOP56 in KRAS-mutant lung cancer cells (H358, H460) by using short-hairpin RNAs (shRNA) (Fig. S2A, B). In contrast to the results from siRNAmediated acute depletion, stable expression of two independent shRNAs showed negligible effects on H358 and H460 proliferation (Fig. S2C, D), which may be due to the activation of compensatory mechanisms. Importantly, several UPR genes, in particular ERN1 and HSPA5 encoding the ER stress sensor IRE1 $\alpha$ and the chaperon protein $\mathrm{BiP}$, respectively, were markedly upregulated in NOP56-depleted H358 and H460 cells (Fig. 2B). Western blot confirmed the increase of BiP, IRE1 $\alpha$, XBP-1s (IRE1 $\alpha$ effector) and of the master UPR transcription factor HSF-1 (heat shock factor 1) and PDI (protein disulfide isomerase), an important ER chaperone induced during ER stress by carrying out a redox reaction and responsible for the formation of disulfide bonds in proteins (Fig. 2C). Notably, p38 MAPK, a key stress-responsive kinase and an UPR effector [37], was highly phosphorylated (activated) in NOP56-depleted H358 cells (Fig. 2C). Moreover, IRE1 $\alpha$ KD blunted p-p38, p-AKT (T308), p-MNK1, p-eIF4E and p-S6 in NOP56-depleted H358 cells, indicating that IRE1 $\alpha$-mediated UPR acts upstream of p38 signaling (Fig. 2D).

Importantly, genetic (siRNA) and pharmacological $(4 \mu 8 \mathrm{C}$, an inhibitor of IRE1 $\alpha)$ inhibition of IRE1 $\alpha$ preferentially impaired NOP56 KD H358 and H460 cells, manifested by significantly greater proliferative inhibition and apoptotic induction in these cells than in control cells (Fig. 2E, F; Fig. S2E, F). Importantly, the increase of IRE1 $\alpha$ KD-induced apoptotic cell death was paralleled by ROS upregulation, and addition of NAC, an ROS scavenger largely dampened IRE1 $\alpha$ KD-induced apoptosis (Fig. 2F, G), supporting a role for the UPR in response to oxidative stress. Similarly, genetic and pharmacological inhibition (with KRIBB11) of HSF1 suppressed the proliferation and evoked apoptotic cell death to a markedly greater extent in NOP56 KD H358 cells than in control cells (Fig. 2H-J).

The outcome of UPR ranges from adaptation to apoptosis [38] and, as such, perturbations of ER homeostasis in cells with an already high level of ER stress, e.g., treatment with bortezomib and tunicamycin that induce persistent ER stress by targeting the $26 \mathrm{~S}$ proteasome and the ER chaperone BiP, respectively, evoke programmed cell death [38, 39]. Indeed, NOP56 KD H358 and H460 cells with high basal levels of ROS are highly susceptible to bortezomib and Tunicamycin compared to control cells (Fig. 2K, L).

Thus, targeting NOP56 disrupts ROS homeostasis and induces IRE1 $\alpha$-mediated UPR in KRAS-mutant lung cancer cells.

\section{IRE1 a-mediated UPR fuels mTOR signaling via p38 MAPK}

To identify cellular processes that may present therapeutic vulnerabilities in NOP56 KD cells, we performed synthetic lethal chemical screens with small-molecule drugs $(n=22)$ that interrogate various oncogenic pathways, with the ER stress inducers (bortezomib and HA15) included as positive controls (Table S2). Our screens showed that, except for bortezomib and HA15, LY294002, AZD5363, and rapamycin, inhibitors of the PI3K/AKT/mTOR pathway, preferentially suppressed

\footnotetext{
(See figure on next page.)

Fig. 2 NOP56 depletion evokes IRE1 a-mediated UPR. A, NOP56 depletion led to significant enrichment of the UPR gene signature in KRAS-mutant cancer cells. GSEA was based on the GEO dataset GSE15212. B, Transcriptional quantification (qRT-PCR) of UPR genes in H358 and H460 cells expressing control (sh Scram) or NOP56-specific shRNA (sh NOP56a). C, Immunoblots of H358 and H460 cells expressing scrambled control or NOP56-specific shRNAs. D, H358 cells expressing scrambled control or NOP56-specific shRNAs were transfected with IRE1a-specific or control siRNAs for $72 \mathrm{~h}$ before immunoblotting. E, Clonogenic assay of $\mathrm{H} 460$ and $\mathrm{H} 358$ cells expressing scrambled control or NOP56-specific shRNAs after treated

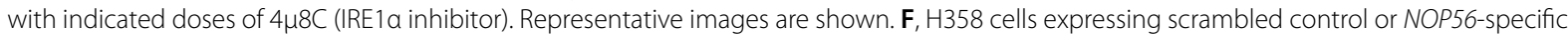
shRNAs were transfected with IRE1 a-specific or control siRNAs for $72 \mathrm{~h}$, in the presence or absence of NAC (2.5 mM) before apoptosis assay. Data are presented as mean $\pm \mathrm{SD}(n=3) .{ }^{* *} P<0.001,{ }^{* * *} P<0.0001$ and ns $P>0.05$ by two-way ANOVA with Tukey's multiple comparisons test. G, H358 cells expressing scrambled control or NOP56-specific shRNAs were transfected with IRE $1 \mathrm{a}$-specific or control siRNAs for $72 \mathrm{~h}$, in the presence or absence of NAC (2.5 mM). Cells were then washed, incubated with H2DCFDA for $30 \mathrm{~min}$, and analyzed by flow cytometry. Quantification of relative ROS levels was shown in the right. Data are presented as mean $\pm \mathrm{SD}(n=3)$. ${ }^{* *} P<0.001,{ }^{* * *} P<0.0001$ and $n s P>0.05$ by two-way ANOVA with Tukey's multiple comparisons test. $\mathbf{H}, \mathrm{H} 358$ cells expressing scrambled control or NOP56-specific shRNAs were transfected with HSF1-specific or control siRNAs for $72 \mathrm{~h}$ before apoptotic assay. I, H358 cells expressing scrambled control or NOP56-specific shRNAs were transfected with HSF1-specific or control siRNAs for $72 \mathrm{~h}$ before immunoblot analysis. Data are presented as mean $\pm \mathrm{SD}(n=3)$. ${ }^{* * *} P<0.001,{ }^{* * * *} P<0.0001$ and ns $P>0.05$ by two-way ANOVA with Tukey's multiple comparisons test. J, Clonogenic assay of H460 and H358 cells expressing scrambled control or NOP56-specific shRNAs after treated with the indicated doses of KRIBB11 (HSF1 inhibitor). Representative images are shown. K, L, Clonogenic assay of H460 and H358 cells expressing scrambled control or NOP56-specific shRNAs after treated with the indicated doses of the ER stress inducer bortezomib (K) or tunicamycin (L). Representative images are shown
} 


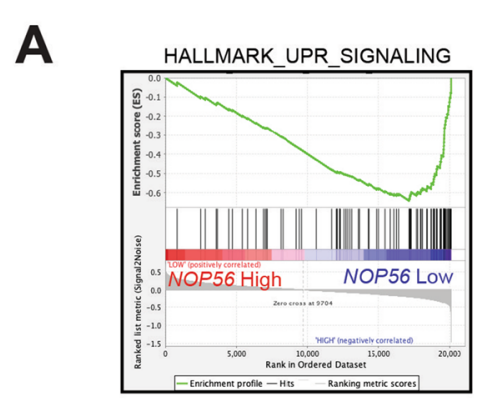

C
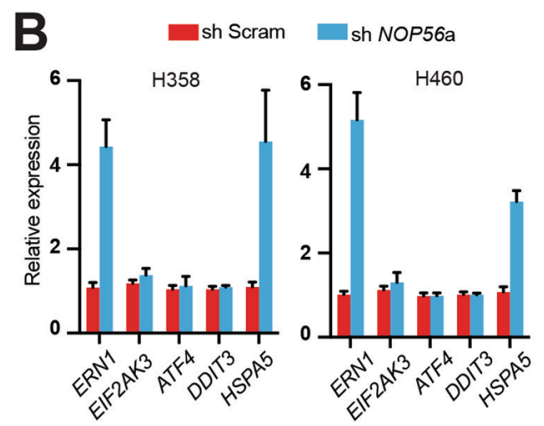
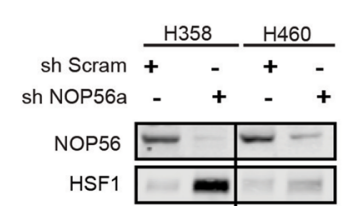

PDI

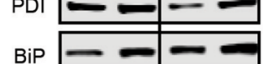

\begin{aligned} & $\operatorname{BiP} \square-\infty \\ &$ PERK $=-\infty \\ &$\hline\end{aligned}

elF2 $\alpha \square$

p-elF $2 \alpha$

IRE1

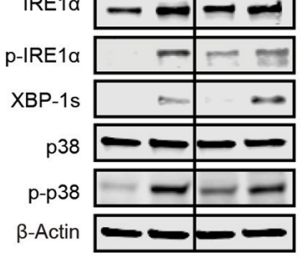

D

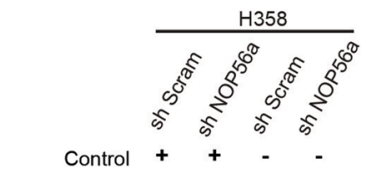

SIRNA IRE $1 \alpha-\quad-\quad+\quad+$

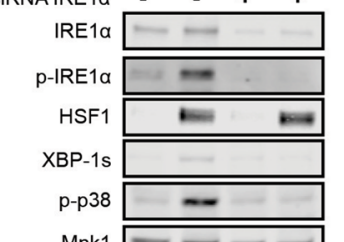

Mnk1 $=\pi=$

p-Mnk1

(T197/202)

p-elF4E

(S209)

p-AKT
(T308)

$\mathrm{p}-\mathrm{S} 6$

(S235/236)

$\beta$-Actin
E
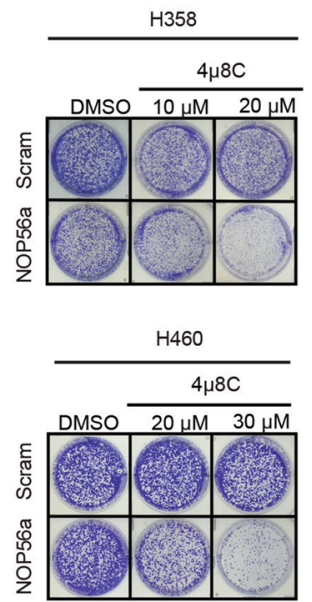

$\mathbf{F}$

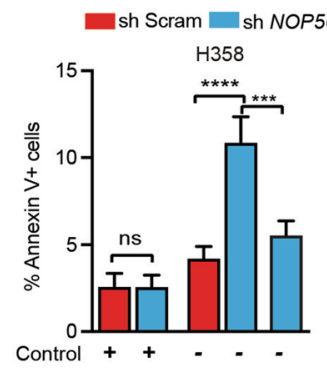

G
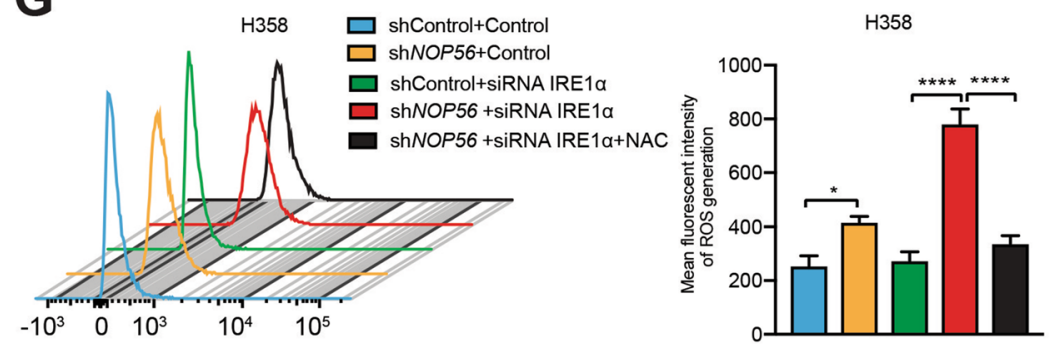

SIRNA IRE1 $\alpha$ - -+++

NAC - - - - +
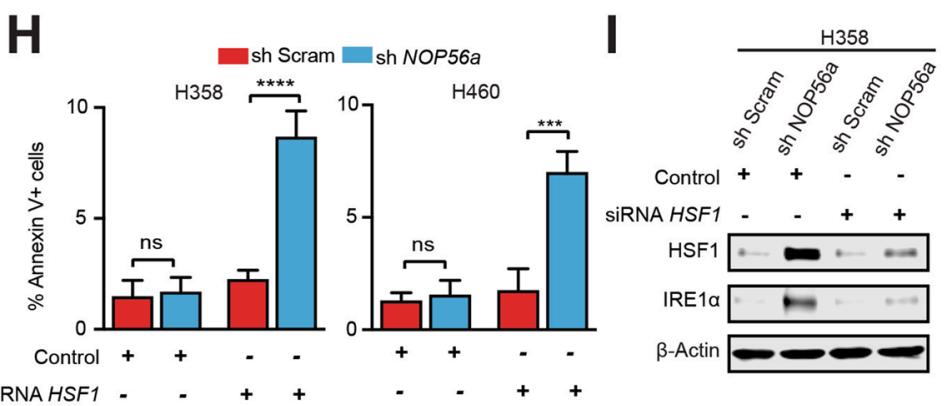

J

K
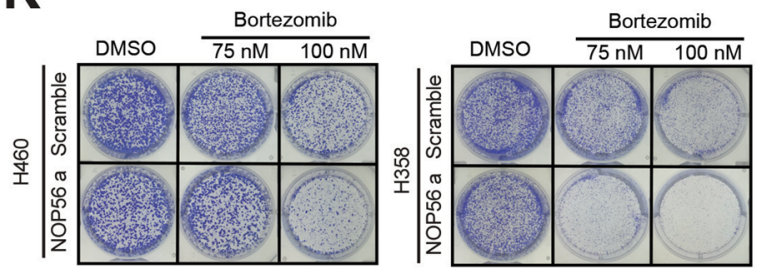

$\mathbf{L}$
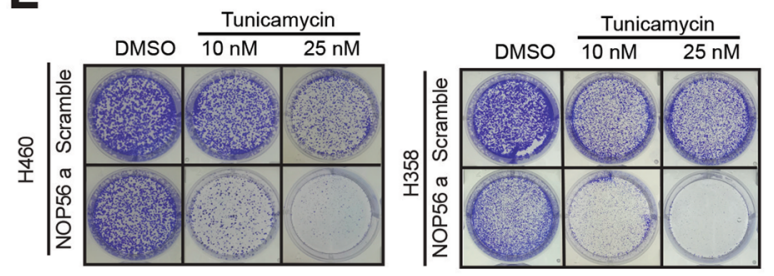

Fig. 2 (See legend on previous page.) 
the viability of NOP56 KD cells, gauged by their $\mathrm{IC}_{50}$ decrease in NOP56 KD H358 and H460 cells versus control cells (Fig. 3A; Fig. S3A). The greatest change in sensitivity was conferred by rapamycin, which was $0.7 \mu \mathrm{M}$ and $1.0 \mu \mathrm{M}$ in H358_shNOP56a and H358_shNOP56b but $12.0 \mu \mathrm{M}$ in H358_Scr cells, with a selectivity index ( $\mathrm{IC}_{50}$ in control cells / $\mathrm{IC}_{50}$ in NOP56 KD cells) of 17 - and 12-fold, respectively (Fig. 3A; Fig. S3A). These observations were validated by independent assays, in which NOP56 KD sensitized H358 and H460 cells to PI3K/ AKT inhibitors (LY294002, AZD5363), anti-mTOR drugs (rapamycin, everolimus), and ER stress inducers (bortezomib and HA15) (Fig. 3B,C; Fig. S3B,C). Importantly, CRISPR/Cas9-mediated knockout of NOP56 dramatically increased the sensitivity of KRAS-mutant (H358, H460) but not of wild-type (H520, H1703) lung cancer cells to rapamycin (Fig. S3D, E).

Moreover, examining gene expression data of KRASmutant cancer cells [36] revealed that NOP56 silencing significantly enriched the mTOR gene signature (Fig. 3D). Mining TCGA and Genomics of Drug Sensitivity in Cancer (GDSC) databases showed that NOP56 expression is negatively correlated with that of mTOR pathway genes in patients with KRAS-mutant lung adenocarcinomas (Fig. 3E) and that NOP56 mRNA levels are a predictive marker of sensitivity $\left(\mathrm{IC}_{50}\right)$ to rapamycin in $K R A S$-mutant cancer cell lines but not in KRAS-wild-type cancer cells (Fig. 3F). These data support our in vitro results (Fig. 3AC; Fig. S3A-C) and further suggest a reciprocal interplay between NOP56 and mTOR signaling.

Indeed, siRNA-mediated NOP56 KD, which slightly increased ribonucleolar proteins (e.g., NOP58, FBL), markedly induced AKT/mTOR (p-AKT, p-mTOR, pS6), translation initiation (p-eIF4E) and the stress-responsive p38 MAPK in H358 cells in a time-dependent manner (Fig. 3G), as did shRNA-mediated stable NOP56
KD in H358 and H460 cells, but not in KRAS-WT lung cancer H1703 cells (Fig. 3H; Fig. S3G). Importantly, NOP56 KD sensitized KRAS-mutant lung (A549), colon (HCT-116, DLD-1, and LS174T), pancreatic (MIAPaCa, HPAF-II) and primary KRAS-mutant lung cancer cells (PF563, PF139) to rapamycin, as well as NRAS-mutant lung cancer $\mathrm{H} 1299$ cells but not KRAS-wild-type $\mathrm{H} 2405$ (BRAF-mutant), EBC-1 (EGFR-mutant), H1993 (MET amplification) and H520 (FGFR1 amplification) cells (Fig. $\mathrm{S} 3 \mathrm{H}, \mathrm{I})$. These results reinforce the notion that NOP56 plays a unique role in KRAS-mutant cancer.

Our results demonstrated that NOP56 and the IRE1 $\alpha$ mediated UPR act upstream of p38 and mTOR signaling (Fig. 2C, D; Fig. 3G), suggesting a signaling cascade from the UPR to mTOR via p38 MAPK. To confirm this, we targeted p38 with the specific inhibitor SB203580, which, as expected, barely affected the upstream IRE1 $\alpha$ dependent UPR (p-IRE1 $\alpha$, XBP-1 s), but strikingly dampened the MNK-eIF4E axis and mTOR signaling (p-AKT, p-S6) in H358 cells (Fig. 3I). Importantly, SB203580 exposure not only decreased activity of the mTOR pathway but also increased PARP expression and promoted PARP cleavage (Fig. 3I), concurrent with substantially elevated cytotoxicity on NOP56 KD H460 and H358 cells compared to that on control cells (Fig. 3J). Together, these results unravel a signaling cascade from the IRE1 $\alpha$ mediated UPR to p38 MAPK and to mTOR signaling in KRAS-mutant lung cancer upon NOP56 suppression.

\section{Synthetic lethality by targeting NOP56 and mTOR in KRAS-mutant lung cancer}

Our findings that NOP56 KD cells are exposed to higher levels of metabolic ROS and display a greater dependency on UPR-activated mTOR signaling suggest a synthetic lethal vulnerability in KRAS-mutant cancer (Figs. 1, 2, 3). To test this hypothesis, we treated NOP56 KD H358

\footnotetext{
(See figure on next page.)

Fig. 3 IRE1 a-mediated UPR fuels mTOR signaling via p38 MAPK. A, H358 cells expressing scrambled control or NOP56-specific shRNAs were treated with different inhibitors, with bar graphs illustrating sensitivity increase after NOP56 KD. Fold changes of IC $C_{50}$ values were presented as $I C_{50}$ of rapamycin in $\mathrm{H} 358$ cells expressing scrambled shRNA (sh_Scrambled) compared to that in H358 cells expressing NOP56-targeted shRNAs (shNOP56 a/b). Data presented as mean ( $n=2$ ). B, Clonogenic assay of H358 and H460 cells expressing control shRNA (sh_Scr) or NOP56-specific shRNAs (sh-a, sh-b) after treatment with indicated doses of rapamycin or everolimus. Representative images are shown. The heatmap (right) indicates the percentage of viable cells after the treatment, based on quantification of clonogenic results (left). Data are presented as mean \pm SD $(n=3)$. C, Growth inhibition of H358 and H460 cells expressing NOP56-specific shRNAs (sh NOP56a, sh NOP56b) or control shRNAs (sh Scram) after treated for $72 \mathrm{~h}$ with the mTOR inhibitors (rapamycin, everolimus). Data are presented as mean $\pm \mathrm{SD}(n=3)$. D, NOP56 silencing significantly enriched the mTOR gene signature in KRAS-mutant cancer cells. GSEA was performed based on the GEO dataset GSE15212. E, Negative correlation of NOP56 mRNA levels with mTOR gene signature (mTOR pathway score) as determined in a TCGA cohort of patients with KRAS-mutant lung adenocarcinoma. Pearson and Spearman coefficient, as well as the significance ( $p$-value), were determined using R software (Cor.test function). F, NOP56 expression is a predictive marker of sensitivity $\left(I C_{50}\right)$ to rapamycin in KRAS-mutant cancer cell lines $(n=18)$ but not in KRAS-wide-type cancer cell lines $(n=82)$. Drug response profiles were downloaded from the GDSC (Genomics of Drug Sensitivity in Cancer) database. G, Immunoblots of H358 cells after transfection with scramble control siRNAs (si-Control) for $72 \mathrm{~h}$ (-) or NOP56-specific siRNAs (si-NOP56) for different time points ( $24 \mathrm{~h}, 48 \mathrm{~h}, 72 \mathrm{~h}$ and 96 h). $\mathbf{H}$, Immunoblots of H358 and H460 cells expressing scrambled control or NOP56-specific shRNAs. I, Immunoblots of H358 cells expressing scramble control or the NOP56-specific shRNAs after treated with the p38 inhibitor SB203580 (5 $\mu \mathrm{M})$ for $24 \mathrm{~h}$. J, Clonogenic assay of H460 and H358 cells expressing control or NOP56-specific shRNAs after treated with the p38 inhibitor SB203580. Representative images are shown
} 


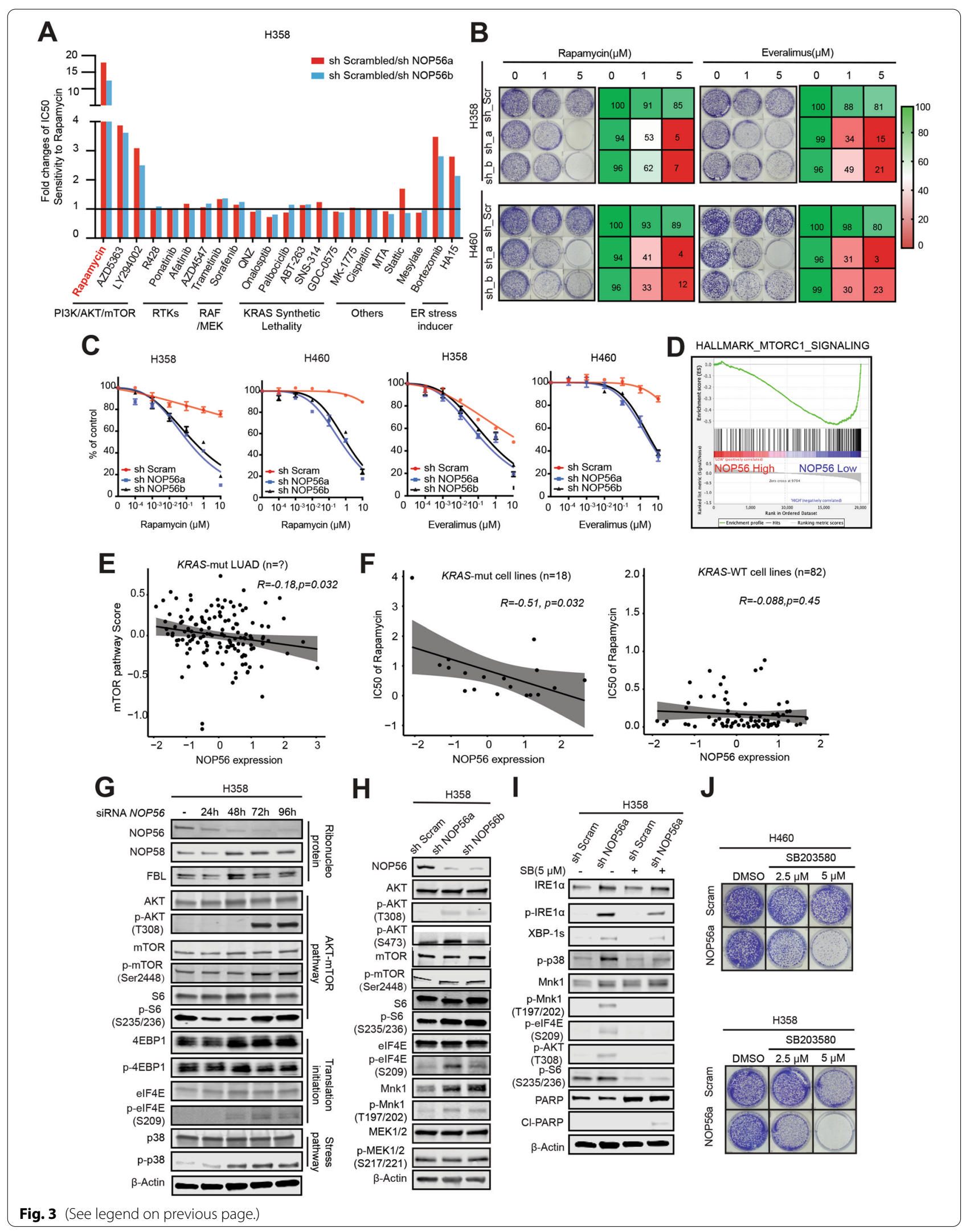


and control cells with rapamycin, which, as expected, decreased mTOR effectors (e.g., p-S6, p-eIF4E) (Fig. 4A) that were the otherwise adaptively upregulated upon NOP56 depletion (Fig. 3G, H). Strikingly, rapamycin induced PARP cleavage (Cl PARP) in NOP56 KD H358 but not in control cells (Fig. 4A), indicating that concomitant targeting of NOP56 and mTOR caused synthetic lethality. Similar results were observed in NOP56 KD H358 cells that were treated with the AKT inhibitor AZD5363 (Fig. S4A, B). Knockdown of Raptor and Rictor, key components of the mTORC1 and mTORC2, respectively, significantly suppressed the viability of NOP56 KD H358 cells despite to differential extent (Fig. S4C, D). Moreover, whereas individual S6 and eIF4E only partly contributed to the viability of NOP56 KD H358 cells, concomitant inhibition of S6 (siRNA) and eIF4E (siRNA and Briciclib, an eIF4E inhibitor) led to significantly enhanced anti-proliferative effect (Fig. 4B-G; Fig. S4E) and largely recapitulated the impact seen by mTOR inhibition with rapamycin (Fig. 4A, Fig. S4F), gauged by the extent to which apoptotic markers (CI-PARP) were induced in NOP56 KD H358 cells (Fig. 4F). These results interrogate an important role for mTOR to relay the IRE1 $\alpha$-mediated UPR signaling in NOP56-depleted $K R A S$-mutant lung cancer.

The UPR is a double-edged sword, as its outcome flips from adaption to apoptosis when malfunctional UPR, a condition at which stress stimuli are overwhelming or the UPR signal cannot be properly relayed $[38,39]$. We thus assumed that the observed synthetic lethality of NOP56 and mTOR inhibition might be enabled due to malfunctional UPR. Indeed, co-targeting NOP56 and mTOR resulted in synergistic effects that not only increased the expression of IRE1 $\alpha$ and $\mathrm{p}$-IRE1 $\alpha$, indicative of hyperactive UPR signals, but also upregulated p-JNK, FOXO3A and BIM, a BH3 only protein and key mediator of apoptotic balance (Fig. 4H). Consistent with their pro-apoptotic roles, this increase of the JNK-FOXO3a-BIM axis was accompanied by PARP cleavage (Cl-PARP) and significantly greater apoptotic cell death in NOP56 KD H358 and H460 cells compared to control cells (Fig. 4H, I). Importantly, IRE1 $\alpha \mathrm{KD}$ (siRNA) precluded the cytotoxicity of combined NOP56 and mTOR inhibition, evidenced by decreased levels of p-JNK, FOXO3a, BIM, Cl-PARP and of apoptotic populations in NOP56 KD H358 and H460 treated with rapamycin (Fig. 4H, I). Similarly, inhibiting JNK activity by the inhibitor SP600125 dampened the efficacy of rapamycin in NOP56 KD H358 and H460 cells (Fig. 4J). Thus, IRE1 $\alpha$-mediated UPR activates mTOR, which provides a survival signal for NOP56 KD KRAS-mutant cancer cells; conversely, mTOR inhibition leads to overwhelmed UPR and promotes apoptotic cell death by activating the JNK-FOXO3A-BIM axis (Fig. 4K).

\section{NOP56 and mTOR converge on a metabolic liability in KRAS-mutant lung cancer}

Next, we asked if the synthetic lethality of co-targeting NOP56 and mTOR is a result of unresolvable metabolic stress. Indeed, rapamycin sensitivity of NOP56 KD H358 and $\mathrm{H} 460$ cells was highly correlated with ROS levels (Fig. 5A, B), and ROS scavenge by NAC significantly compromised the cytotoxicity of rapamycin (Fig. 5B), highlighting a causative link between ROS and rapamycin-induced apoptosis in NOP56 KD cells.

These results uncover a novel homeostatic mechanism of metabolic stress mediated by NOP56 and validate an unexpected synthetic lethality by targeting NOP56 and mTOR that aggregate a metabolic liability in KRASmutant lung cancer (Fig. 5C).

\section{NOP56 downregulation plus rapamycin potently suppresses in vivo tumor growth of KRAS-mutant lung cancer}

Finally, we investigated in vivo efficacy of co-targeting NOP56 and mTOR. In a xenograft model from KRASmutant H460 cells, NOP56 knockdown (shNOP56) only mildly inhibited tumor growth compared to control

\footnotetext{
(See figure on next page.)

Fig. 4 Synthetic lethality by targeting NOP56 and mTOR in KRAS-mutant lung cancer cells. A, Immunoblots of H358 cells expressing scramble control or NOP56-specific shRNAs after treated with rapamycin (1 $\mathrm{MM}$ ) for $24 \mathrm{~h}$. B-G H358 cells expressing scramble control or shNOP56-specific shRNAs were transfected with control siRNAs or the indicated siRNAs specifically targeting S6, elF4E, alone and in combination. The cells were then subjected to immunoblots (B, D, F) and viability assay (C, E, G) $72 \mathrm{~h}$ post-transfection. Data are presented as mean \pm SD ( $n=3$ ). $\mathbf{H}, \mathrm{H} 358 \mathrm{Cells}$ expressing scrambled control or NOP56-specific shRNAs were transfected with IRE1a-specific or control siRNAs for $48 \mathrm{~h}$, followed by treatment with rapamycin $(1 \mu \mathrm{M})$ for $24 \mathrm{~h}$ before immunoblotting. I, H358 cells expressing control or NOP56-specific shRNAs were transfected with IRE 1 a-specific or control siRNAs for $24 \mathrm{~h}$, followed by treatment with rapamycin $(5 \mu \mathrm{M})$ for $72 \mathrm{~h}$ before apoptosis assay. Data are presented as mean \pm SD $(n=3) .{ }^{*} p<0.05,{ }^{* *} p<0.001$ and ${ }^{* * * *} p<0.0001$ by two-way ANOVA with Tukey's multiple comparisons test. J, H358 cells expressing control or NOP56-specific shRNAs were preincubated overnight with vehicle (DMSO) or the JNK inhibitor SP600125, followed by treatment with rapamycin for $72 \mathrm{~h}$ before apoptosis assay. Data are presented as mean $\pm \mathrm{SD}(n=3) .{ }^{* *} p<0.01,{ }^{* * *} P<0.001$ and ns $P>0.05$ by two-way ANOVA with Tukey's multiple comparisons test. K, Proposed model of cellular gauge for IRE1a-regulated UPR. In KRAS-mutant cancer cells, intact NOP56 keeps ROS in check so that IRE1 a-regulated UPR is minimal (basal level; left). Intermediate levels of IRE1 a-regulated UPR ensue from NOP56 depletion, which activates p38-AKT/mTOR and promotes cell survival (middle). At "dangerous" level of ROS, IRE1a-regulated UPR initiates JNK-dependent apoptosis (right)
} 

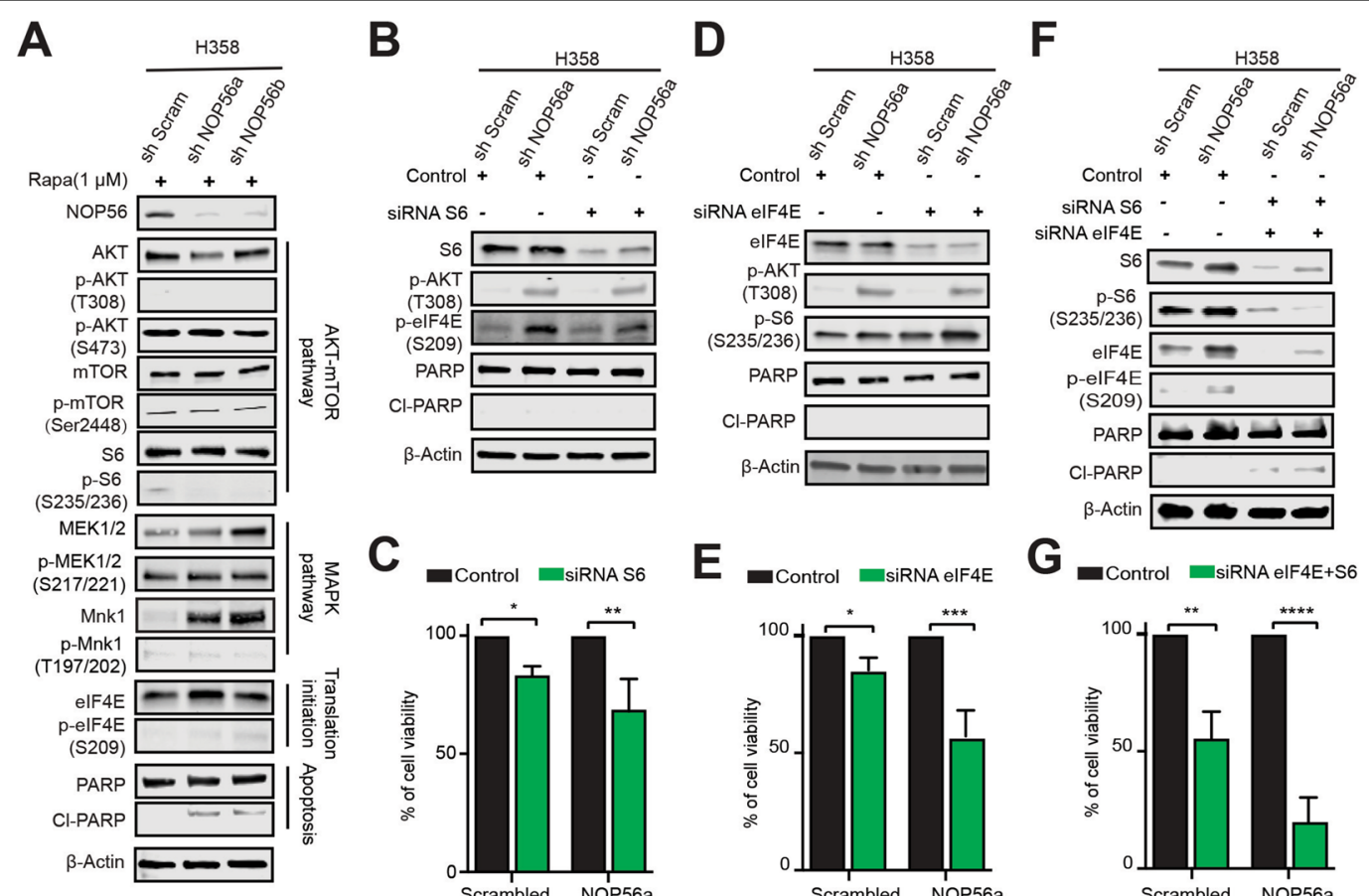
SIRNA elF4E - - + +
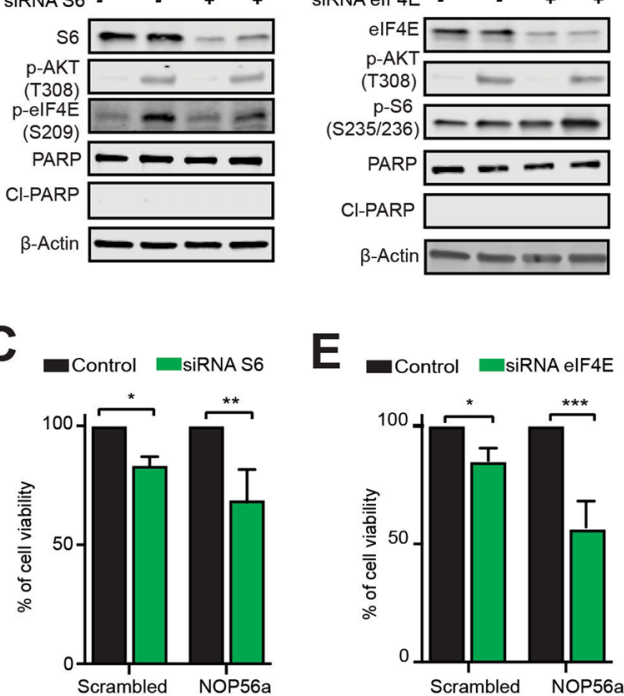

E

G
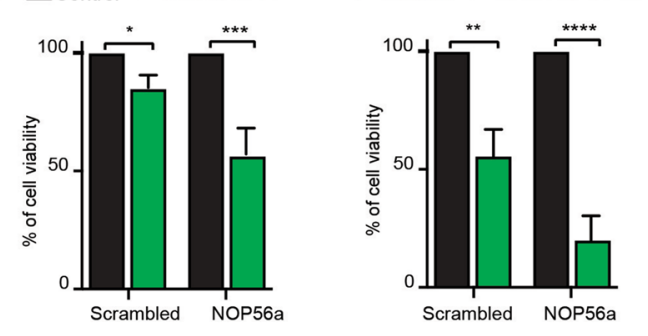

H

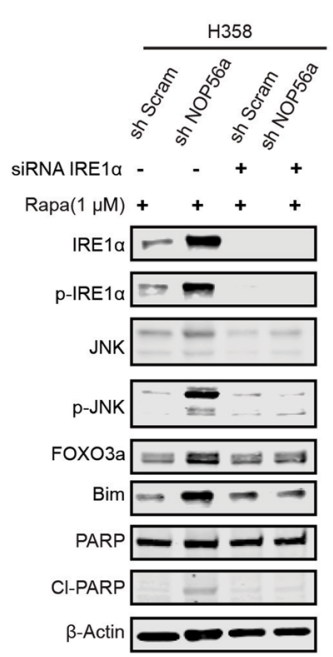

I

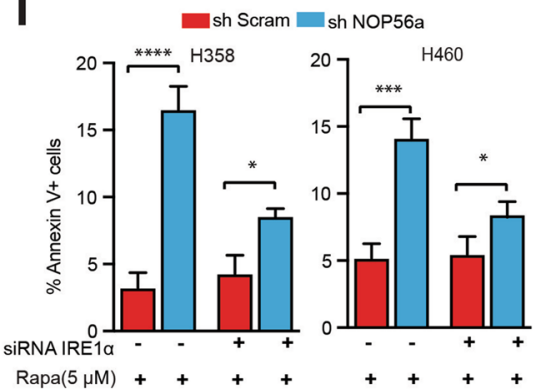

K
J
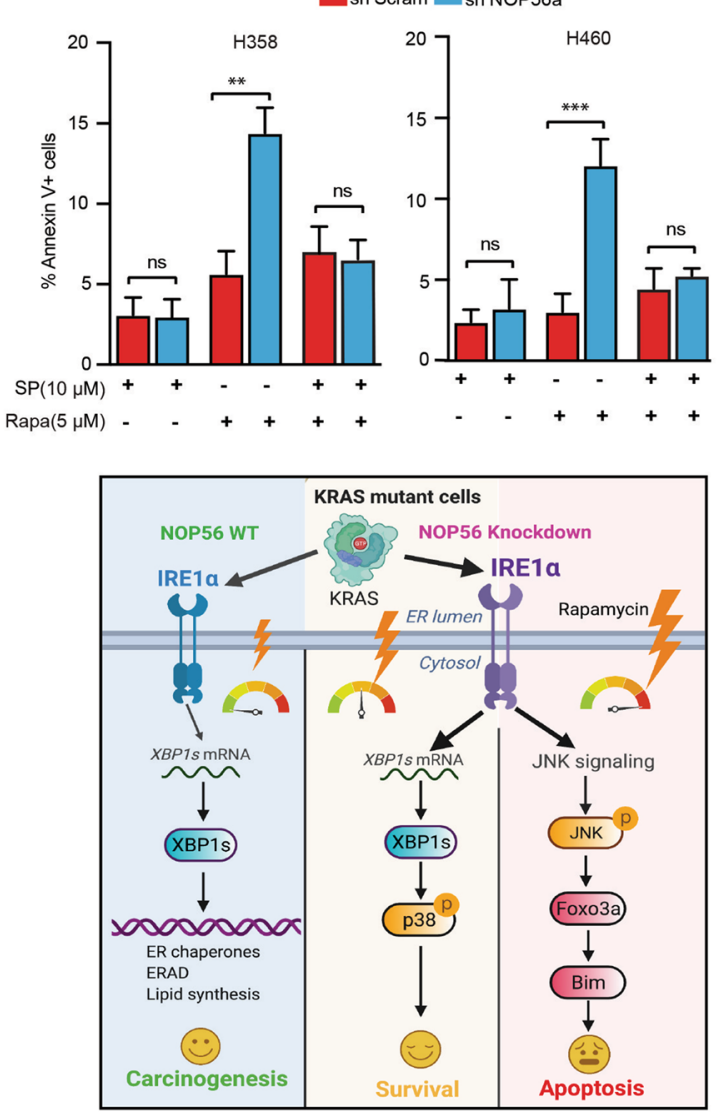

Fig. 4 (See legend on previous page.) 


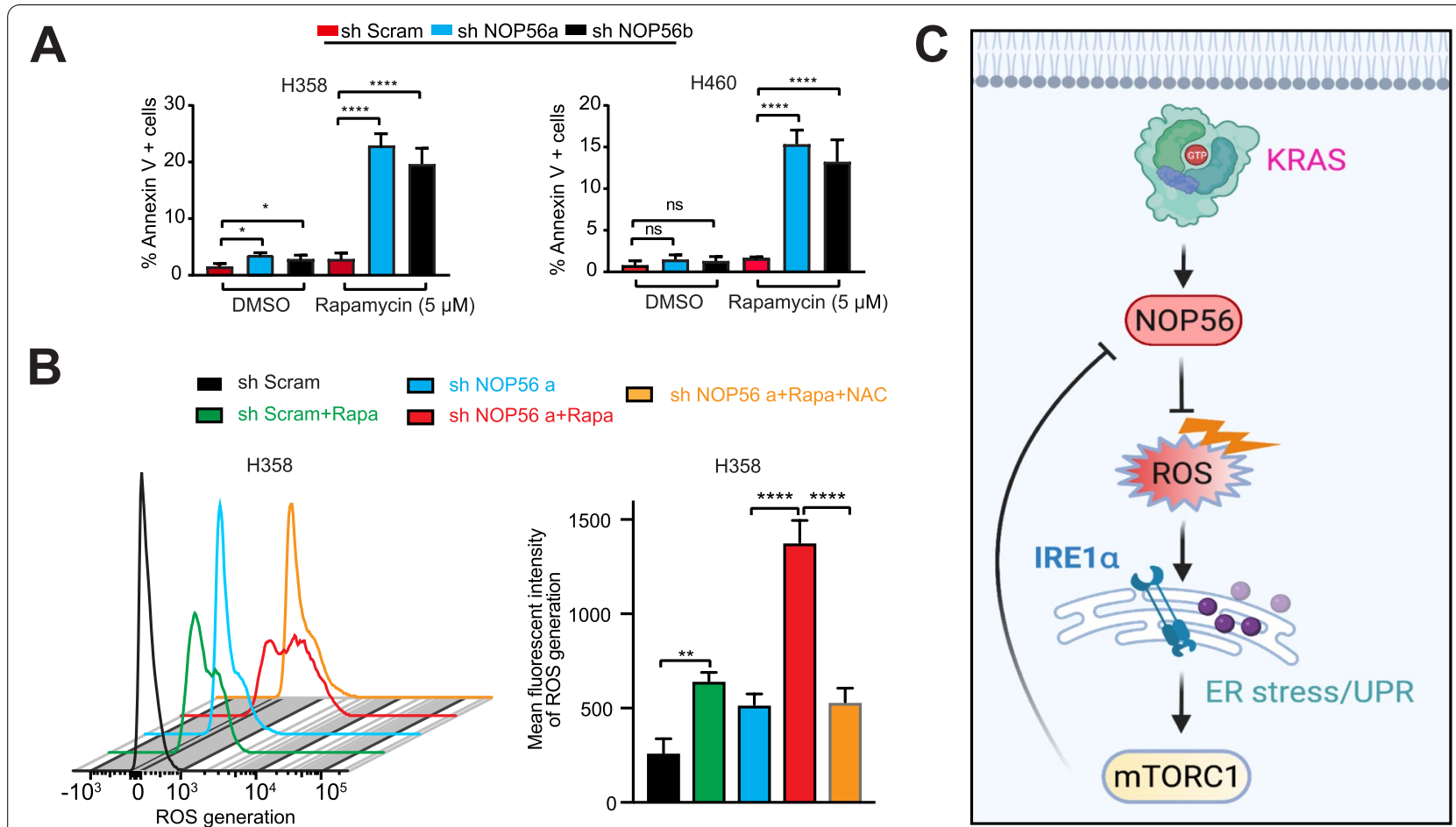

Fig. 5 NOP56 and mTOR converge on a metabolic liability in KRAS-mutant tumor growth. A, Apoptosis assay of H358 and H460 cells expressing control or NOP56-specific shRNAs after treatment with rapamycin $(5 \mu \mathrm{M})$ for $72 \mathrm{~h}$. Data are presented as mean of three independent experiments $(n=3) .{ }^{*} p<0.05,{ }^{* * *} P<0.0001$ and ns $P>0.05$ by two-way ANOVA with Tukey's multiple comparisons test. B, H358 and H460 cells expressing control or NOP56-specific shRNAs were treated with rapamycin $(5 \mu \mathrm{M})$ for $24 \mathrm{~h}$. Cells were then washed, incubated with H2DCFDA for 30 min, and analyzed by flow cytometry. Quantification of relative ROS levels was shown in the right. C, A working model for the function of NOP56 in KRAS-mutant cancers

shRNA (shScrambled), as did rapamycin (Fig. 6A). However, the outcome of concomitant targeting of NOP56 and mTOR (shNOP56 plus rapamycin) was superior to that achieved by shNOP56 or rapamycin alone, leading to far more effective and potent suppression of xenograft tumor growth (Fig. 6A, B). Residual tumors (after 3-week treatment) from the combination group (shNOP56 plus rapamycin) were typically tiny and significantly differed from those of the other treatment groups (Fig. 6C). Immunohistochemical
(IHC) analysis revealed that the anti-tumor efficacy of combined treatment with shNOP56 and rapamycin was paralleled by marked decrease of mTOR activity (p-AKT, p-mTPOR, p-S6) and increase in apoptosis (Caspase-3) in the residual tumors (Fig. S5A).

Similar results were obtained from H358 xenografts (Fig. 6D, E) and a patient-derived xenograft (PDX) model established from the primary KRAS-mutant PF139 cells (Fig. 6F-I). In both models, NOP56 KD sensitized H358 and PF139 xenograft tumors to rapamycin, leading to

(See figure on next page.)

Fig. 6 NOP56 knockdown plus rapamycin inhibits KRAS-mutant tumor growth. A, Growth curve of xenograft tumors derived from H460 cells expressing either a control or an shRNA against NOP56 (shNOP56a). Rapamycin $(0.1 \mathrm{mg} / \mathrm{kg}$ ) was administrated i.p. for 3 weeks ( 5 days $/$ week). Data are shown as mean $\pm \mathrm{SD}){ }^{* * *} P<0.001,{ }^{*} P<0.05$ and $\mathrm{ns}(P>0.05)$ by two-way ANOVA with Tukey's multiple comparisons test. B, Relative tumor volume of $\mathrm{H} 460$ xenograft tumors after the treatment for 3 weeks. C, Weights of $\mathrm{H} 460$ xenograft tumors after the treatment for 3 weeks. ${ }^{* * *} P<0.001$ by one-way ANOVA with Tukey's multiple comparisons test. D. Growth curve of xenograft tumors derived from H358 cells expressing either a control or an shRNA against NOP56 (shNOP56a). ${ }^{*} P<0.01$ by two-way ANOVA with Tukey's multiple comparisons test. E, Kaplan-Meier survival curve of mice with $\mathrm{H} 358$ xenografts from the experiment in D. F, Growth curve of PDX tumors derived from primary KRAS-mutant PF139 lung cancer cells expressing either control or NOP56-specific shRNAs. Data are shown as mean \pm SD). ${ }^{* *} P<0.001$ and ${ }^{*} P<0.05$ by two-way ANOVA with Tukey's multiple comparisons test. Immunoblots of PF139 cells expressing NOP56-specific or scrambled shRNAs was also shown. G, Relative tumor volume of PF139 xenografts after 3 weeks of treatment. $\mathbf{H}$, Weights of PF139 xenograft tumors after treated for 3 weeks. ${ }^{* *} P<0.001,{ }^{*} P<0.05$ and ns $P>0.05$ by two-way ANOVA with Tukey's multiple comparisons test. I, H\&E and IHC of p-AKT(T308), p-mTOR(S2448), p-S6(S235/236), Ki67 and Caspase-3) in PF139 xenograft tumors after the treatment. Scale bars $100 \mu \mathrm{m}$ 


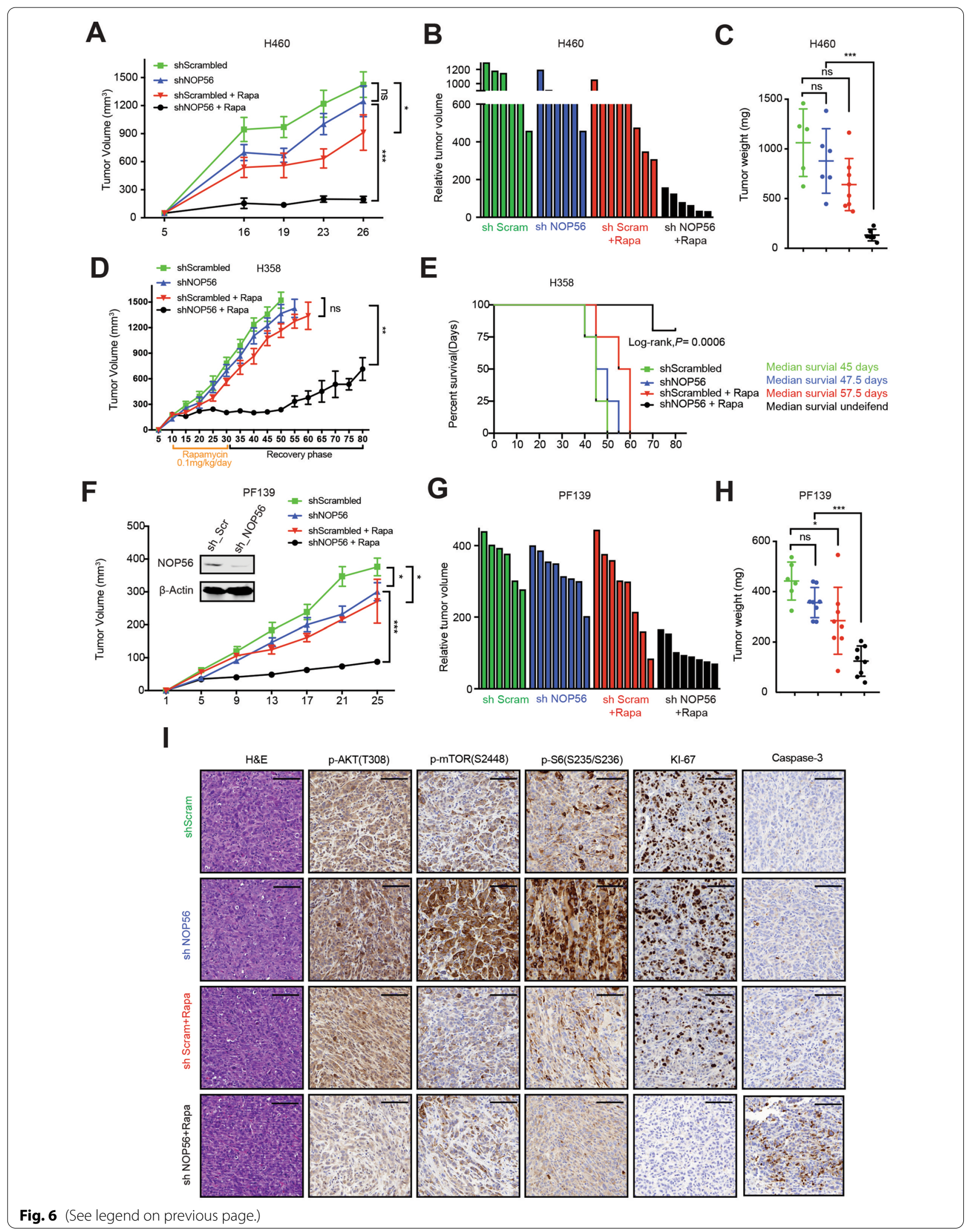


potent suppression of tumor growth (Fig. 6D, F-H) and significant improvement of mouse survival (Fig. 6E). IHC of the PF139 residual tumors revealed that NOP56 depletion plus rapamycin strikingly suppressed tumor cell proliferation (Ki-67) and dampened mTOR activity (p-AKT, p-mTOR and p-S6), but increased Caspase-3 cleavage (Fig. 6I), which is consistent with the in vitro results (Figs. 4, 5) and our observations on H460 xenografts (Fig. S5A). Notably, rapamycin showed little beneficial effects in NOP56 KD KRAS wild-type H1703 xenografts (Fig. $\mathrm{S} 5 \mathrm{~B}, \mathrm{C}$ ), which mirrors the in vitro data and reinforces the selective activity of co-targeting NOP56/mTOR in $K R A S$-mutant lung cancer.

Together, these results support a model that NOP56 downregulation induces a metabolic vulnerability to mTOR inhibition, which presents a new and rational strategy for treating $K R A S$-mutant lung cancer.

\section{Discussion}

In the present study, we have uncovered a new and unanticipated mechanism by which NOP56 and mTOR signaling cooperate in metabolic stress response in $K R A S$-mutant lung cancer. We show that NOP56 suppresses ROS, and its depletion induces synthetic lethal susceptibility to inhibition of mTOR that is otherwise essential for counterbalance of the resurge of cytotoxic ROS evoked by NOP56 downregulation. We also discover that mTOR activation is driven by IRE $1 \alpha$-mediated UPR via 38 MAPK. These findings support a model that NOP56 plays a role in the surveillance of ROS homeostasis and suggest that concomitant blockage of NOP56 and mTOR signaling has the potential to selectively target $K R A S$-mutant lung cancer. As multiple mTOR inhibitors (e.g., rapamycin and everolimus) are clinically approved drugs, our observations have immediate translational significance.

Despite decades-long steady efforts, therapeutic targeting of KRAS-mutant cancers has remained an overarching challenge in clinical oncology [3]. A promising strategy to target $K R A S$-driven tumors is to exploit cancer cell vulnerabilities contextually co-opted by mutant KRAS, in light of the concept that mutant KRAS alter physiological biochemical networks and induces cellular stresses, rendering $K R A S$-mutant cancer particularly susceptible to inhibition of stress-remedy mechanisms [12-14]. Empowered by CRISPR- and shRNA-based functional genomics, a plethora of novel factors required for KRAS-mutant cancer cells have been identified [12$16,26]$, although the long-sought-after universal synthetic lethal targets for KRAS-driven pan-cancers are still at large. By implementing integrated analysis of functional genomic datasets $(n=5)$ derived from shRNAand CRISPR-based screens [12, 16, 26], we revealed that NOP56 confers a metabolic requirement for $K R A S$-mutant cancer by regulating ROS homeostasis. A functional link between NOP56 and mutant KRAS is supported by a multitude of lines of evidence, i.e., the elevated expression of NOP56 in KRAS-mutant tumors, the prognostic significance of NOP56 expression in patients with KRAS-mutant but not wild-type cancers, and selective damage on KRAS-mutant cancer cells incurred by NOP56 downregulation.

Moreover, our results uncover a reciprocal interaction of NOP56 and mTOR signaling and suggest that combined inhibition of NOP56/mTOR is a rational strategy to combat KRAS-mutant cancer. Supporting our findings, mRNA levels of NOP56 significantly correlate with that of mTOR pathway genes in KRAS-mutant cancer cells and lung adenocarcinomas, and NOP56 expression is a predictive marker of sensitivity to mTOR inhibitors in KRAS-mutant but not KRAS-wild-type cancer cells. Importantly, NOP56 knockdown sensitizes KRASmutant cancer cells to mTOR inhibitors in vitro and in vivo, which is not true for $K R A S$-wild-type tumor cells. Despite potential toxicological challenges as ribosome biogenesis is also an important physiological process, modulation of NOP56 activity may afford a therapeutic window for targeted inhibition of mTOR in KRASmutant cancers.

An increasingly growing body of evidence suggests that oncogenic KRAS signaling rewires metabolic pathways to meet the energetic and biosynthetic demands of cancer cells $[27-30,40]$. In particular, increased ROS production, which has been shown to be functionally required for KRAS-mediated tumorigenicity [27, 28], is a key metabolic manifestation associated with $K R A S$-mutant cancer cells [27-30]. Since excess ROS is harmful, cancer cells must leverage ROS levels to favor tumor progression but prevent cell death $[14,27-30,41]$. Here, we reported, for the first time, a role for NOP56 in metabolic ROS response in $K R A S$-mutant lung cancer. NOP56 is a key component of box $\mathrm{C} / \mathrm{D}$ snoRNPs that regulates ribosome assembly. This process has been shown to be deregulated in tumors with increased requirement for protein synthesis, providing cancer vulnerabilities for therapeutic avenues [21-24, 42-45]. Our results are also in line with previous studies reporting cancer subtype-specific alterations in ribosome assembly and biogenesis processes [44, 45]. In addition, recent evidence suggests that snoRNPs may also be involved in other processes independent of their functions in ribosome biogenesis [46]. Future studies will be necessary to clarify whether the newly identified metabolic role of NOP56 in KRAS-mutant cancer is related to its canonical role.

Our finding that IRE1 $\alpha$-mediated UPR connects the NOP56 function in ROS scavenge with mTOR 
signaling, a master regulator of cellular metabolism [47], provides mechanistic insights about the synthetic lethality of co-targeting NOP56 and mTOR, which is supported the observation that challenges to ribosome biogenesis result in acute loss of proteostasis [48]. IRE1 $\alpha$-mediated UPR, whilst initially protective, turns to be pro-apoptotic if ROS-induced metabolic stress is prolonged and persists [38, 39]. We further reveal that the overwhelming metabolic stress incurred by NOP56 depletion and mTOR inhibition activates the JNK-BIM axis, a stress-responsive pathway that promotes cell-cycle arrest and apoptosis [37]. The observation that UPR is a key component of the homeostatic mechanism in response to ROS resurge evoked by NOP56 knockdown is consistent with the notion that UPR plays an import role in homeostasis regulation including ROS and that challenges to ribosome biogenesis result in acute loss of proteostasis [49]. As metabolic ROS is causally linked to mutant KRAS-induced tumorigenicity and requires homeostatic mechanisms to maintain ROS levels within a threshold favorable for tumor development $[14,27-30,41]$, the identification of NOP56 and mTOR converging on a role as ROS scavengers reveals an unanticipated metabolic vulnerability in KRAS-mutant cancers.

\section{Conclusion}

In summary, we have uncovered an unexpected role for NOP56 in the surveillance of metabolic ROS in $K R A S$-mutant lung cancer. We have also revealed a novel synthetic lethality between NOP56 depletion and mTOR inhibitors that occurs by impeding the homeostatic mechanism of ROS in KRAS-mutant cancer cells. Moreover, we have demonstrated that mTOR activation upon NOP56 depletion is driven by IRE1 $\alpha$ mediated UPR. These results shed light on the mechanisms underlying KRAS-induced metabolic rewiring, reveals an unanticipated metabolic vulnerability in $K R A S$-mutant lung cancer, and suggest a new rationale for the treatment of the disease. Because KRAS alterations are implicated in a broad spectrum of human malignancies, our findings may also be applicable to other lineages of cancer with high frequencies of $K R A S$ alterations.

\footnotetext{
Abbreviations

GSEA: Gene Set Enrichment Analysis; IRE1a: Inositol-requiring enzyme 1a; KRAS: Kirsten rat sarcoma viral oncogene homolog; mTOR: Mechanistic target of rapamycin; NOP56: Nucleolar protein 5A; PARP: Poly (ADP-ribose) polymerase; RNAi: RNA interference; ROS: Reactive oxygen species; TCGA: The Cancer Genome Atlas; UPR: Unfolded protein response.
}

\section{Supplementary Information}

The online version contains supplementary material available at https://doi. org/10.1186/s13046-022-02240-5.

Additional file 1: Figure S1. NOP56 knockdown inhibits proliferation of KRAS-mutant cancer cells. A, Immunoblots of KRAS-mutant and KRAS-wild type cancer cells that were transfected with NOP56-specific siRNAs (siNOP56) or scramble control siRNAs (si-Control). B, KRAS mutant and KRAS wild type cancer cells were transfected with control siRNAs or NOP56specific siRNAs. Cell viability was determined $72 \mathrm{~h}$ post transfection. Data are presented as mean $\pm \mathrm{SD}(n=3) .{ }^{*} p<0.05,{ }^{* *} p<0.01,{ }^{* * *} p<0.001$ ${ }^{* * *} P<0.0001$ and ns $P>0.05$ by two-way ANOVA with Tukey's multiple comparisons test. C, NOP56 is not a biomarker of survival in patients with KRAS-wild-type lung adenocarcinoma (LC), pancreatic cancer (PC) and colon cancer (CC). Kaplan-Meier survival analyses of patient cohorts in TCGA were stratified by the optimal cut-off value of the mRNA level of NOP56. D, Gene set enrichment analysis (GSEA) of a TCGA cohort of patients with KRAS-mutant lung $(n=141)$, pancreatic $(n=133)$ and colon cancer $(n=170)$. Figure S2. Stable expression of NOP56-specific shRNAs activates IRE1 a-mediated UPR. A, Immunoblots of H358 and H460 cells expressing scrambled control or NOP56 shRNAs. B, Immunofluorescence of $\mathrm{H} 358$ and $\mathrm{H} 460$ cells that express scrambled control or NOP56 shRNAs. The NOP56 signal is indicated by arrowheads. C, The cell viability curve of H358 and H460 cells expressing scramble control shRNA or the NOP56targeted shRNAs was measured at the indicated time points. D, Clongenic assay of $\mathrm{H} 358$ and $\mathrm{H} 460$ cells expressing scramble control or NOP56-targeted shRNAs. Quantification of clongenic assay were shown underneath. Data are presented as mean \pm SD $(n=3)$. E, Growth inhibition of H358 and $\mathrm{H} 460$ cells expressing control shRNA or NOP56-targeted shRNA (3000 cells/well) treated for $72 \mathrm{~h}$ with the indicated doses of an IRE1 a inhibitor $(4 \mu 8 \mathrm{C})$. Data are presented as mean $\pm \mathrm{SD}(n=3)$. $\mathbf{F}$, Apoptosis assay of H460 cells expressing scrambled control or NOP56-targeted shRNAs after transfection with IRE $1 a$-specific or control siRNAs for $72 \mathrm{~h}$. Data are presented as mean $\pm \mathrm{SD}(n=3) .{ }^{* *} P<0.001$ and $n s P>0.05$ by two-way ANOVA with Tukey's multiple comparisons test. Figure S3. NOP56 KD renders KRAS-mutant lung cancer cells susceptible to mTOR inhibition. A, Bar graphs illustrating the change of sensitivity to different inhibitors in H460 cells after NOP56 knockdown. Data are presented as $I C_{50}$ values of the indicated inhibitors in $\mathrm{H} 460$ cells expressing scramble control shRNAs compared to $\mathrm{IC}_{50}$ in $\mathrm{H} 460$ cells expressing NOP56-targeted shRNAs. Data are shown as mean $(n=2)$. B, Viability assay of $\mathrm{H} 460$ and $\mathrm{H} 358$ cells expressing control shRNA or NOP56-targeted shRNA (3000 cells/well) after treated for $72 \mathrm{~h}$ with the indicated doses of PI3K inhibitor (LY294002) and AKT inhibitor (AZD5363). Data are presented as mean \pm SD $(n=3)$. C, Viability assay of $\mathrm{H} 460$ and $\mathrm{H} 358$ cells expressing control shRNA or NOP56-targeted shRNA (3000 cells/well) after treated for $72 \mathrm{~h}$ with the indicated doses of BiP inhibitor (HA15) and ER stress inducer (bortezomib). Data are presented as mean \pm SD $(n=3)$. $\mathbf{D}$, Immunoblots of KRAS-mutant (H358, H460) and wild-type (H1703, H520) cells expressing NOP56-specific sgRNAs. E, Viability assay of the cells expressing control or NOP56specific sgRNAs after treated with rapamycin for $72 \mathrm{~h}$. Data are shown as mean \pm SD $(n=3)$. F, NOP56 is negatively correlated with PI3K/AKT/ mTOR pathway genes (PI3KCA, PDPK1, PIK3R1) in KRAS-mutant lung cancer patients. Pearson and Spearman coefficient and significance ( $p$-value) are analyzed using R software (Cor.test function). G, Immunoblots of H460 and H1703 cells expressing control or NOP56-targeted shRNAs. H, I, Viability assay of KRAS-mutant $(\mathbf{H})$ and wildtype (I) cancer cells expressing control or NOP56-specific siRNAs after treated with rapamycin. The assay was performed $72 \mathrm{~h}$ after drug treatment ( $96 \mathrm{~h}$ after siRNA transfection). Figure S4. NOP56 KD activates and induces dependence on the mTOR pathway in KRAS-mutant cancer cells. A, Immunoblots of H358 cells expressing control or NOP56-targeted shRNAs after treated with the AKT inhibitor (AZD5363) for 24h. B, Clongenic assay of H358 cells expressing control or NOP56-specific shRNAs after treated with indicated doses of AZD5363. Representative images are shown. C, D, Immunoblots (C) and viability assay (D) of H358 cells expressing control or NOP56-specific shRNAs after transfected with raptor- or rictor-specific or control siRNAs for $72 \mathrm{~h}$. Data are presented as mean $\pm \mathrm{SD}(n=3)$. $\mathbf{E}$, Clongenic assay of H358 and $\mathrm{H} 460$ cells expressing control shRNA or NOP56-specific shRNAs after 
treatment with indicated doses of elF4E inhibitor (Briciclib). Representative images are shown. F, Immunoblots of $\mathrm{H} 460$ cells expressing control or NOP56-target shRNAs after treated with rapamycin $(1 \mu \mathrm{M})$ for $24 \mathrm{~h}$. Figure S5. In vivo activity and selectivity of co-targeting NOP56 and mTOR in KRAS-mutant lung cancer. A, H\&E and IHC analysis of p-AKT(T308), p-mTOR(S2448),p-S6(S235/236), Ki67 and Caspase-3) in residual H460 xenograft tumors after the indicated treatment. Scale bars $100 \mu \mathrm{m}$. B Tumor volume of $\mathrm{H} 1703$ xenografts in immunocompromised (NSG) mice. H1703 cells were transduced with either a control or an shRNA against NOP56 (shNOP56a). Tumors were measured every 5 days with a caliper. C, Kaplan-Meier survival curve of mice harboring $\mathrm{H} 1703$ xenografts from the experiment shown in B.

Additional file 2: Table S1. Cell lines used in this study. Table S2. Inhibitors used for synthetic lethal chemical screens. Table S3. Antibodies used in this study. Table S4.KRAS synthetic lethal (SL) genes.

\section{Acknowledgements}

We gratefully acknowledge Christelle Dubey (Division of Thoracic Surgery, Inselspital, Bern University Hospital) for technical support, especially with animal studies and CRISPR-based knockout of NOP56. We thank the West-German Biobank Essen (WBE) for the collaboration in establishment of the PF139 and PF526 lung cancer cells. The Translational Research Unit at the Institute of Pathology, University of Bern is acknowledged for assistance of IHC staining.

\section{Authors' contributions}

ZY, SQL designed and performed the experiments, analyzed the data and wrote the manuscript. LZ, HY performed the experiments and analyzed the data. TMM, BH, YG, BZ, CC and WW analyzed the data and edited the manuscript. PD and GJK provided conceptual inputs, analyzed the data and edited the manuscript. RAS provided financial support and edit the manuscript. RWP conceived the project, supervised the study and wrote the paper. All authors read and approved the final version of the manuscript.

\section{Funding}

This study was supported by a grant from Swiss National Science Foundation (SNSF \#310030_192648; to R-W. Peng) and PhD fellowships from China Scholarship Council (ZY, LZ, YG).

\section{Availability of data and materials}

All data generated or analysed during this study are included in this published article and its supplementary information files.

\section{Declarations}

\section{Ethics approval and consent to participate}

The establishment of PF139 and PF526 cells was approved by the Ethics Committee of the University Hospital Essen (\#18-8208-BO), Germany, with written consents obtained from the patients. The study was performed in accordance with the Declaration of Helsinki. Mouse studies were approved by the Veterinary Office of Canton Bern, Switzerland, and conducted in accordance with Institutional Animal Care.

\section{Consent for publication}

Not applicable.

\section{Competing interests}

The authors declare no competing interests.

\section{Author details}

'Division of General Thoracic Surgery and Department of BioMedical Research (DBMR), Inselspital, Bern University Hospital, University of Bern, Murtenstrasse 28, 3008 Bern, Switzerland. ${ }^{2}$ Current address: University of Massachusetts Medical School, Worcester, MA 01605, USA. ${ }^{3}$ Current address: Department of Thoracic Surgery, Shanghai Chest Hospital, Shanghai Jiao Tong University, Shanghai 200030, China. ${ }^{4}$ Department of Thoracic Surgery, University Medicine Essen - Ruhrlandklinik, University Duisburg-Essen, Essen, Germany. ${ }^{5}$ Department of Thoracic surgery, Fujian Medical University Union Hospital, Fuzhou City, Fujian, China. ${ }^{6}$ Thoracic Surgery Department 2, Hunan Cancer
Hospital and The Affiliated Cancer Hospital of Xiangya School of Medicine, Central South University, Changsha, Hunan, China.

Received: 23 May 2021 Accepted: 1 January 2022

Published online: 17 January 2022

\section{References}

1. Prior IA, Lewis PD, Mattos C. A comprehensive survey of Ras mutations in cancer. Cancer Res. 2012;72(10):2457-67.

2. Reck M, Rabe KF. Precision diagnosis and treatment for advanced nonsmall-cell lung Cancer. N Engl J Med. 2017;377(9):849-61.

3. Yang $\mathrm{H}$, Liang SQ, Schmid RA, Peng RW. New horizons in KRAS-mutant lung cancer: dawn after darkness. Front Oncol. 2019;9:953.

4. Jeanson A, Tomasini P, Souquet-Bressand M, Brandone N, Boucekine $M$, Grangeon $M$, et al. Efficacy of immune checkpoint inhibitors in KRAS-mutant non-small cell lung Cancer (NSCLC). J Thorac Oncol. 2019;14(6):1095-101.

5. Janes MR, Zhang J, Li LS, Hansen R, Peters U, Guo X, et al. Targeting KRAS mutant cancers with a covalent G12C-specific inhibitor. Cell. 2018;172(3):578-89.

6. Molina-Arcas M, Moore C, Rana S, van Maldegem F, Mugarza E, RomeroClavijo P, et al. Development of combination therapies to maximize the impact of KRAS-G12C inhibitors in lung cancer. Sci Transl Med. 2019;11(510):eaaw7999.

7. Ostrem JM, Peters U, Sos ML, Wells JA, Shokat KM. K-Ras(G12C) inhibitors allosterically control GTP affinity and effector interactions. Nature. 2013;503(7477):548-51.

8. Manchado E, Weissmueller S, Morris JP 4th, Chen CC, Wullenkord R, Lujambio A, et al. A combinatorial strategy for treating KRAS-mutant lung cancer. Nature. 2016;534(7609):647-51.

9. Shimizu T, Tolcher AW, Papadopoulos KP, Beeram M, Rasco DW, Smith LS, et al. The clinical effect of the dual-targeting strategy involving PI3K/AKT/ mTOR and RAS/MEK/ERK pathways in patients with advanced cancer. Clin Cancer Res. 2012;18(8):2316-25.

10. Huang A, Garraway LA, Ashworth A, Weber B. Synthetic lethality as an engine for cancer drug target discovery. Nat Rev Drug Discov. 2020;19(1):23-38.

11. Downward J. RAS synthetic lethal screens revisited: still seeking the elusive prize? Clin Cancer Res. 2015;21(8):1802-9.

12. Luo J, Emanuele MJ, Li D, Creighton CJ, Schlabach MR, Westbrook TF, et al. A genome-wide RNAi screen identifies multiple synthetic lethal interactions with the Ras oncogene. Cell. 2009;137(5):835-48.

13. De Raedt T, Walton Z, Yecies JL, Li D, Chen Y, Malone CF, et al. Exploiting cancer cell vulnerabilities to develop a combination therapy for rasdriven tumors. Cancer Cell. 2011;20(3):400-13.

14. Yang Z, Liang SQ, Saliakoura M, Yang H, Vassella E, Konstantinidou G, et al. Synergistic effects of FGFR1 and PLK1 inhibitors target a metabolic liability in KRAS-mutant cancer. EMBO Mol Med. 2021;13(9):e13193.

15. Martin TD, Cook DR, Choi MY, Li MZ, Haigis KM, Elledge SJ. A role for mitochondrial translation in promotion of viability in K-Ras mutant cells. Cell Rep. 2017;20(2):427-38.

16. Kim J, McMillan E, Kim HS, Venkateswaran N, Makkar G, Rodriguez-Canales J, et al. XPO1-dependent nuclear export is a druggable vulnerability in KRAS-mutant lung cancer. Nature. 2016;538(7623):114-7.

17. Nagel R, Semenova EA, Berns A. Drugging the addict: non-oncogene addiction as a target for cancer therapy. EMBO Rep. 2016;17(11):1516-31.

18. Solimini NL, Luo J, Elledge SJ. Non-oncogene addiction and the stress phenotype of cancer cells. Cell. 2007;130(6):986-8.

19. Gautier T, Berges T, Tollervey D, Hurt E. Nucleolar KKE/D repeat proteins Nop56p and Nop58p interact with Nop1p and are required for ribosome biogenesis. Mol Cell Biol. 1997;17(12):7088-98.

20. Newman DR, Kuhn JF, Shanab GM, Maxwell ES. Box C/D snoRNA-associated proteins: two pairs of evolutionarily ancient proteins and possible links to replication and transcription. RNA. 2000;6:861-79.

21. Cowling VH, Turner SA, Cole MD. Burkitt's lymphoma-associated c-Myc mutations converge on a dramatically altered target gene response and implicate Nol5a/Nop56 in oncogenesis. Oncogene. 2014;33(27):3519-27. 
22. Su H, Xu T, Ganapathy S, Shadfan M, Long M, Huang TH, et al. Elevated snoRNA biogenesis is essential in breast cancer. Oncogene. 2014;33:1348-58.

23. Gong J, et al. A Pan-cancer analysis of the expression and clinical relevance of small nucleolar RNAs in human Cancer. Cell Rep. 2017;21(7):1968-81.

24. Pelletier J, Thomas G, Volarevic S. Ribosome biogenesis in cancer: new players and therapeutic avenues. Nat Rev Cancer. 2018;18(1):51-63.

25. Bohnsack KE, Bohnsack MT. Uncovering the assembly pathway of human ribosomes and its emerging links to disease. EMBO J. 2019;38:e100278.

26. Wang T, Yu H, Hughes NW, Liu B, Kendirli A, Klein K, et al. Gene essentiality profiling reveals gene networks and synthetic lethal interactions with oncogenic Ras. Cell. 2017;168:890-903.

27. Weinberg F, Hamanaka R, Wheaton WW, Weinberg S, Joseph J, Lopez M, et al. Mitochondrial metabolism and ROS generation are essential for Kras-mediated tumorigenicity. Proc Natl Acad Sci U S A. 2010;107(19):8788-93.

28. Guo JY, Chen HY, Mathew R, Fan J, Strohecker AM, Karsli-Uzunbas G, et al. Activated Ras requires autophagy to maintain oxidative metabolism and tumorigenesis. Genes Dev. 2011;25(5):460-70.

29. Yun J, Mullarky E, Lu C, Bosch KN, Kavalier A, Rivera K, et al. Vitamin C selectively kills KRAS and BRAF mutant colorectal cancer cells by targeting GAPDH. Science. 2015;350(6266):1391-6.

30. Storz P. KRas, ROS and the initiation of pancreatic cancer. Small GTPases. 2017;8(1):38-42.

31. Yang Z, Liang SQ, Yang H, Xu D, Bruggmann R, Gao Y, et al. CRISPRmediated kinome editing prioritizes a synergistic combination therapy for FGFR1-amplified lung cancer. Cancer Res Cancer Res. 2021;81(11):3121-33.

32. Liang SQ, Buhrer ED, Berezowska S, Marti TM, Xu D, Froment L, et al. mTOR mediates a mechanism of resistance to chemotherapy and defines a rational combination strategy to treat KRAS-mutant lung cancer. Oncogene. 2019;38(5):622-36.

33. Yang H, Liang SQ, Xu D, Yang Z, Marti TM, Gao Y, et al. HSP90/AXL/elF4Eregulated unfolded protein response as an acquired vulnerability in drug-resistant KRAS-mutant lung cancer. Oncogenesis. 2019;8(9):45.

34. El Hassouni B, Sarkisjan D, Vos JC, Giovannetti E, Peters GJ. Targeting the ribosome biogenesis key molecule Fibrillarin to avoid Chemoresistance. Curr Med Chem. 2019;26(33):6020-32.

35. Marcel V, Ghayad SE, Belin S, Therizols G, Morel AP, Solano-Gonzalez E, et al. p53 acts as a safeguard of translational control by regulating fibrillarin and rRNA methylation in cancer. Cancer Cell. 2013;24(3):318-30.

36. Grade M, Hummon AB, Camps J, Emons G, Spitzner M, Gaedcke J, et al. A genomic strategy for the functional validation of colorectal cancer genes identifies potential therapeutic targets. Int J Cancer. 2011;128(5):1069-79.

37. Darling NJ, Cook SJ. The role of MAPK signalling pathways in the response to endoplasmic reticulum stress. Biochim Biophys Acta. 1843;2004:2150-63.

38. Hetz C, Zhang K, Kaufman RJ. Mechanisms, regulation and functions of the unfolded protein response. Nat Rev Mol Cell Biol. 2020;21(8):421-38.

39. Xu D, Liang SQ, Yang H, Lüthi U, Riether C, Berezowska S, et al. Increased sensitivity to apoptosis upon endoplasmic reticulum stress-induced activation of the unfolded protein response in chemotherapy-resistant malignant pleural mesothelioma. Br J Cancer. 2018;1 19(1):65-75.

40. Cantor JR, Sabatini DM. Cancer cell metabolism: one hallmark, many faces. Cancer Discov. 2012;2(10):881-98.

41. DeNicola GM, Karreth FA, Humpton TJ, Gopinathan A, Wei C, Frese K, et al. Oncogene-induced Nrf2 transcription promotes ROS detoxification and tumorigenesis. Nature. 2011;475(7354):106-9.

42. Bursać S, Prodan Y, Pullen N, Bartek J, Volarević S. Dysregulated ribosome biogenesis reveals therapeutic liabilities in Cancer. Trends Cancer. 2021;7(1):57-76.

43. Bustelo XR, Dosil M. Ribosome biogenesis and cancer: basic and translational challenges. Curr Opin Genet Dev. 2018;48:22-9.

44. Justilien V, Ali SA, Jamieson L, Yin N, Cox AD, Der CJ, et al. Ect2-dependent rRNA synthesis is required for KRAS-TRP53-driven lung adenocarcinoma. Cancer Cell. 2017;31(2):256-69.

45. Zhou F, Liu Y, Rohde C, Pauli C, Gerloff D, Köhn M, et al. AML1-ETO requires enhanced C/D box snoRNA/RNP formation to induce selfrenewal and leukaemia. Nat Cell Biol. 2017;19(7):844-55.
46. Albert B, Kos-Braun IC, Henras AK, Dez C, Rueda MP, Zhang X, et al. A ribosome assembly stress response regulates transcription to maintain proteome homeostasis. Elife. 2019:8:e45002.

47. Mossmann D, Park S, Hall MN. mTOR signalling and cellular metabolism are mutual determinants in cancer. Nat Rev Cancer. 2018;18(12):744-57.

48. Tye BW, Commins N, Ryazanova LV, Wühr M, Springer M, Pincus D, et al. Proteotoxicity from aberrant ribosome biogenesis compromises cell fitness. Elife. 2019;8:e43002.

49. Metcalf MG, Higuchi-Sanabria R, Garcia G, Tsui CK, Dillin A. Beyond the cell factory: Homeostatic regulation of and by the UPR ${ }^{E R}$. Sci AdV. 2020;6(29):eabb9614.

\section{Publisher's Note}

Springer Nature remains neutral with regard to jurisdictional claims in published maps and institutional affiliations.
Ready to submit your research? Choose BMC and benefit from:

- fast, convenient online submission

- thorough peer review by experienced researchers in your field

- rapid publication on acceptance

- support for research data, including large and complex data types

- gold Open Access which fosters wider collaboration and increased citations

- maximum visibility for your research: over 100M website views per year

At BMC, research is always in progress.

Learn more biomedcentral.com/submissions 